\title{
Species comparison of liver proteomes reveals links to naked mole-rat longevity and human aging
}

\author{
Ivonne Heinze ${ }^{1 \dagger}$, Martin Bens ${ }^{1 \dagger}$, Enrico Calzia ${ }^{2 \dagger}$, Susanne Holtze ${ }^{3}$, Oleksandr Dakhovnik ${ }^{1}$, Arne Sahm', \\ Joanna M. Kirkpatrick', Karol Szafranski ${ }^{1}$, Natalie Romanov ${ }^{4}$, Sai Nagender Sama', Kerstin Holzer ${ }^{5}$, Stephan Singer ${ }^{5}$,

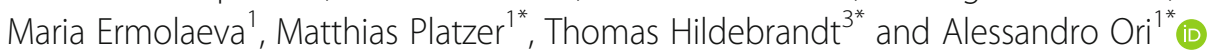

\begin{abstract}
Background: Mammals display a wide range of variation in their lifespan. Investigating the molecular networks that distinguish long- from short-lived species has proven useful to identify determinants of longevity. Here, we compared the livers of young and old long-lived naked mole-rats (NMRs) and the phylogenetically closely related, shorter-lived, guinea pigs using an integrated omics approach.

Results: We found that NMR livers display a unique expression pattern of mitochondrial proteins that results in distinct metabolic features of their mitochondria. For instance, we observed a generally reduced respiration rate associated with lower protein levels of respiratory chain components, particularly complex I, and increased capacity to utilize fatty acids. Interestingly, we show that the same molecular networks are affected during aging in both NMRs and humans, supporting a direct link to the extraordinary longevity of both species. Finally, we identified a novel detoxification pathway linked to longevity and validated it experimentally in the nematode Caenorhabditis elegans.

Conclusions: Our work demonstrates the benefits of integrating proteomic and transcriptomic data to perform cross-species comparisons of longevity-associated networks. Using a multispecies approach, we show at the molecular level that livers of NMRs display progressive age-dependent changes that recapitulate typical signatures of aging despite the negligible senescence and extraordinary longevity of these rodents.
\end{abstract}

\section{Background}

Among mammals, lifespan generally correlates with other life-history parameters such as gestation period and body mass [1]. In this perspective, a subterranean rodent, the naked mole-rat (Heterocephalus glaber, NMR), and humans represent two species outliers by having an exceptionally long lifespan relative to their body mass. NMRs are eusocial animals that live in colonies where only a subgroup of animals is devoted to reproduction (usually a queen and one male called pasha) [2]. NMRs exhibit other exceptional traits

\footnotetext{
* Correspondence: matthias.platzer@leibniz-fli.de; HILDEBRAND@izw-berlin.de; alessandro.ori@leibniz-fli.de

${ }^{\dagger}$ Ivonne Heinze, Martin Bens, and Enrico Calzia contributed equally to this work as co-first authors.

Matthias Platzer, Thomas Hildebrandt, and Alessandro Ori are co-senior authors. ${ }^{1}$ Leibniz Institute on Aging - Fritz Lipmann Institute (FLI), Jena, Germany

3 Leibniz Institute for Zoo and Wildlife Research (IZW), Berlin, Germany

Full list of author information is available at the end of the article
}

including lifelong fertility, resistance to infection, high regenerative capacity, and resistance to cancer and diabetes, reviewed in $[3,4]$. For these reasons, NMRs have drawn attention of multiple studies aimed at identifying the molecular mechanisms behind their extreme longevity and resistance to age-related diseases. Comparative genome analysis has revealed positively selected genes in NMR (including genes involved in cellular respiration, translation, and regulation of oxidative stress) [2, 5-7], and RNA-seq analysis suggested fewer changes in gene expression during aging as compared to other mammals $[6,8]$, supporting the view of enhanced maintenance of homeostasis in NMRs at the molecular level. NMRs possess enhanced protein stability and increased proteasomal activity in the liver $[9,10]$, negligible levels of cellular senescence [11], increased resistance to irradiation-induced senescence and apoptosis [12], over-activation of pathways that contribute to stress resistance (e.g., the nuclear factor 
erythroid2-related factor 2 (NFE2L2, previously NRF2/Nrf2) in the liver and fibroblasts and p53 in fibroblasts) [3, 13], atypical expression of extracellular matrix components by fibroblasts, such as high molecular mass hyaluronan, that confer resistance against cancer development [14]. Intriguingly, NMRs have higher steady-state levels of oxidative damage compared to, e.g., mouse $[9,15]$, and possess mitochondria with unusual morphology in the heart and skeletal muscle [16]. However, NMRs appear to be protected from the age-dependent increase in oxidative damage that manifest in other species [9], presumably due to enhanced detoxifying systems [17].

There is a wealth of evidence linking energy metabolism to the aging process and organism lifespan. Dietary interventions and nutrient sensing pathways have been shown to play a central role in modulating aging in different organisms [18-20]. Studies of genes under positive selection pressure across related species that show different lifespan have highlighted genes involved in mitochondrial homeostasis balance [21], likely influencing both energy metabolism and hormetic responses affecting lifespan [22]. Age-dependent changes of mitochondrial ultrastructure and activity have been described both in flies and mice [23].

There is emerging evidence that metabolic changes in the NMR contribute to adaptation to its ecosystem [24], allow to act as a "superorganism" with its eusocial life style [4], and might be related to its extreme longevity. Given its central role in organism metabolism, we set out to investigate the liver of NMRs in order to identify novel molecular signatures of longevity. Since ecological adaptations are more likely to affect gene expression [25], and mechanisms of aging act both at the transcript and protein level [26], we performed a cross-species comparison between NMR and the shorter-lived guinea pig (Cavia porcellus, GP) using an integrated proteomic and transcriptomic approach. In order to investigate cross-species differences in the context of aging, we additionally analyzed the livers from young and old NMRs and related the identified changes to human aging by studying the liver proteome of 12 individuals aged between 31 and 88 years. Finally, we validated one of the newly identified longevity pathways to be a mediator of lifespan in the nematode Caenorhabditis elegans.

\section{Results}

Cross-species comparison of liver proteomes reveals elevated expression of detoxifying enzymes in naked mole-rats

We first compared the liver proteomes of young adult, non-breeder, male NMRs $(2.7-3.8$ years old $($ yo), $n=4)$ and GPs $(0.7-1$ years old, $n=4)$ using mass spectrometry (Additional file 1: Table S1). We chose GP as the most closely related rodent $(\sim 41$ mya divergence time [7]) that displays a shorter lifespan as compared to NMR (maximum lifespan 12 years for GP vs. 31 years for
NMR [27]) and has a genome sequence available. For each animal, we obtained a quantitative proteome profile by liquid chromatography tandem mass spectrometry and estimated absolute protein abundances using the iBAQ method [28]. In order to directly compare the two species, we mapped both NMR and GP proteins to the respective human orthologs and used these as the reference for comparison (see "Methods" section). This allowed us to perform a quantitative comparison of the two species using estimated absolute abundances for 3248 protein groups quantified by at least two unique proteotypic peptides in both species (Fig. 1a and Additional file 2: Table S2). In order to validate our approach, we obtained RNA-seq data from the same samples and determined transcript-level fold changes between the two species. For this, only those reads that exclusively mapped to conserved regions were used, a method used for transcriptomic cross-species comparisons [8] (Additional file 2: Table S2). Protein and transcript fold changes displayed a significant positive correlation (Pearson $R=0.52, p<2.2 \mathrm{e}-16$, considering all the cross-quantified cases; Pearson $R=0.78, p<2.2 \mathrm{e}-16$, considering all the cases significant in both dataset $(q<0.1)$; Fig. $1 b)$, which is in line with comparisons performed within the same species [29]. Despite the general of trend of consistency, we also found a minority of genes that showed discordant fold changes at the transcript and protein level (Additional file 3: Figure S1A). Taken together, these data indicate that our strategy can reveal meaningful differences in protein abundance between species and that many of these changes are driven by changes in transcript levels.

We next asked whether our comparison would reveal differential abundance of proteins that have been shown to influence lifespan in other organisms. We found strikingly higher levels of peroxiredoxin 1 (PRDX1) and thioredoxin reductase 1 (TXNRD1) in NMRs as compared to GPs (Fig. 1b). PRDX1 and TXNRD1 are both known targets of the transcription factor NFE2L2 [30]. They display the strongest increase in NMR vs. GP among the 28 NFE2L2 target genes significantly affected at both the transcript and protein level $(q<0.1$, corresponding to a combined $q<0.05$, Fig. $1 \mathrm{c}$ and Additional file 2: Table S2). PRDX1 and TXNRD1 play a crucial role in maintaining cell redox homeostasis, and both of them have been shown to influence lifespan in multiple species by buffering ROS and promoting proteostasis [31-33]. Interestingly, PRDX1 and TXNRD1 are cytosolic enzymes, and their mitochondrial counterparts (PRDX5, TXNRD2) are instead expressed at similar levels in both species (Additional file 2: Table S2).

We next wondered whether we could identify similar differences in other organs. For this purpose, we compared RNA-seq data from the heart, skin, kidney, and cerebellum across the two species [34] and found that 

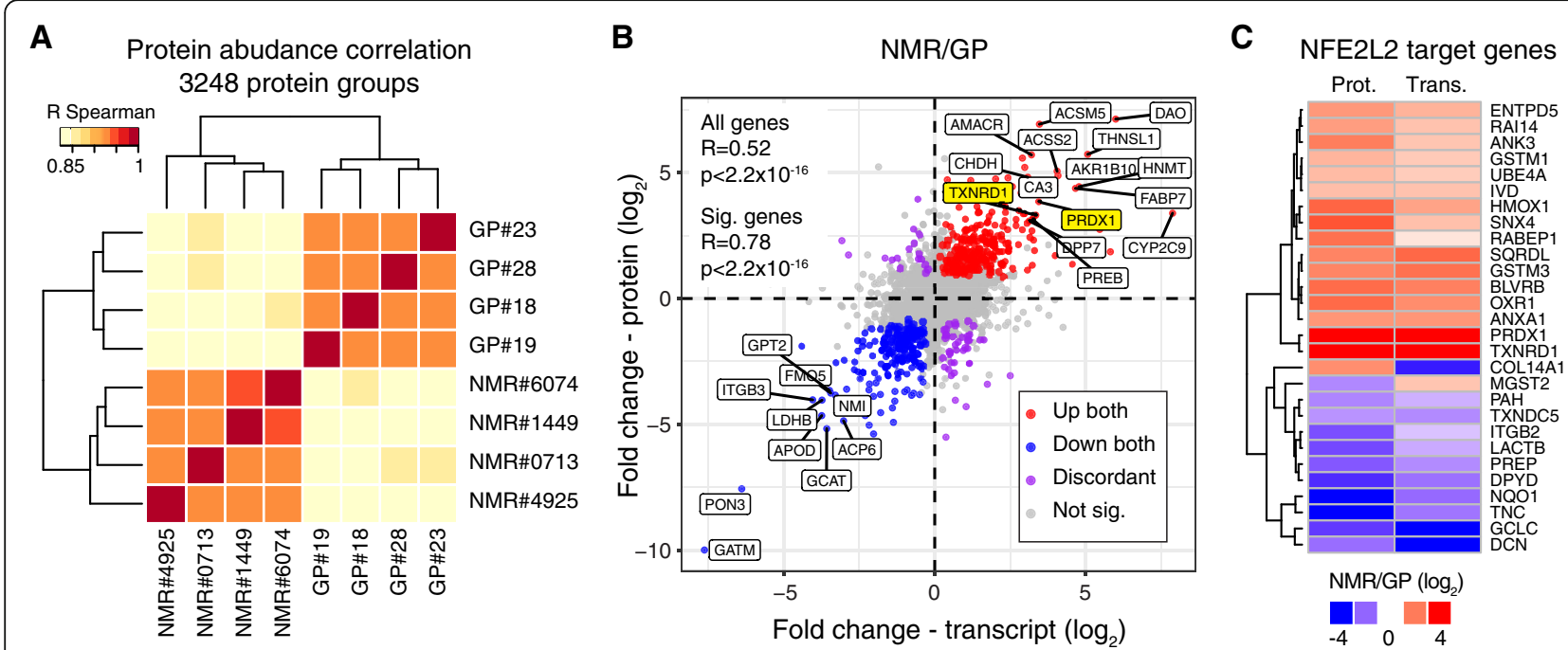

Fig. 1 Cross-species comparison of the liver proteome integrated with RNA-seq from the same animals. a Liver proteomes from four adult naked mole-rats (NMRs) and four guinea pigs (GPs) were compared using quantitative mass spectrometry. Hierarchical clustering based on the correlation between proteome profiles based on 3248 protein groups quantified across the two species. b Comparison of NMR/GP fold changes estimated at the protein level by quantitative mass spectrometry and at the transcript level by RNA-seq. Colored dots indicate genes significant $(q<0.1)$ in both comparisons. The names of selected genes that show consistent fold changes in the two comparisons are indicated. For RNA-seq analysis, only reads mapping to conserved regions between the two species were considered. $\mathbf{c}$ Heatmap showing NFE2L2 target genes significantly affected in NMR vs. GP. List of NFE2L2 basal target genes was obtained from [30]. Only genes significant at both the transcript and protein level $(q<0.1$, corresponding to a combined $q<0.05$ ) are shown. Related to Additional file 1: Table S1, Additional file 2: Table S2, and Additional file 6: Figure S2

increased transcript levels of PRDX1 occur exclusively in the liver (Additional file 3: Figure S1B and S1C). This suggests that increased level PRDX1 and TXNRD1 might be linked to a specific metabolic activity of the NMR liver.

\section{The liver of naked mole-rats has a unique metabolism characterized by reduced mitochondrial respiration and enhanced lipid metabolism}

Intrigued by this finding, we used gene set enrichment analysis to investigate differences in pathways and molecular networks between the long-lived and shorter-lived species. Our analysis returned pathways linked to energy metabolism (Fig. 2a and Additional file 4: Table S3). In particular, we found pathways related to lipid metabolism to be upregulated, and gene sets related to oxidative phosphorylation to be downregulated in NMR $(q<0.05)$. Among upregulated proteins involved in lipid metabolism, we found enzymes responsible for fatty acid beta-oxidation (e.g., ACOX2 and ACOX3), and lipid (e.g., ACACA and ACSL5), cholesterol (e.g., MVD and DHCR24) and bile acids biosynthesis (e.g., AMACR) compared to GP (Fig. 2a, b and Additional file 2: Table S2). Many of these are direct target of the nuclear receptor peroxisome proliferator-activated receptor alpha (PPAR $\alpha$ ), a master regulator of energy metabolism linked to aging [35] (Fig. 2b). The majority of these enzymes showed higher abundance in the liver of NMRs both at transcript and protein level, irrespectively of their sub-cellular localization (Fig. 2b).
In order to exclude that such differences would arise from a different organelle composition of the NMR liver cells, we analyzed the distribution of fold changes for proteins belonging to different cell compartments and found slight, but significantly lower general levels of mitochondrial proteins (mean $\log _{2}$ fold change $=-0.17, p=0.01$, Welch two sample $t$ test), and higher levels of lysosomal proteins (mean $\log _{2}$ fold change $=0.2, p=0.005$, Welch two sample $t$ test) in NMR compared to GP.

Regarding oxidative phosphorylation, NMRs showed reduced abundance of a subset of mitochondrial respiratory chain components (Fig. 2c). Interestingly, these differences manifested almost exclusively at the protein level and affected to different extents the respiratory chain complexes, with components of complex I being the most strongly reduced (mean $\log _{2}$ fold change $=-1.53, p=7.2 \times 10^{-5}$, Welch two sample $t$ test, Fig. 2d). The opposite changes of mitochondrial enzymes involved in lipid metabolism and respiratory chain components indicate that NMR livers possess a distinct composition of their mitochondrial proteome.

In order to investigate whether proteomic differences result in altered mitochondrial activity of NMR liver parenchyma, we performed ex vivo measurements of cellular respiration in liver extracts of NMR by means of high-resolution respirometry (see the "Methods" section). We measured mitochondrial respiration from an independent group of young ( 1 year old, $n=4$ ) and middle-aged (10 years old, $n=4)$ male NMRs and 


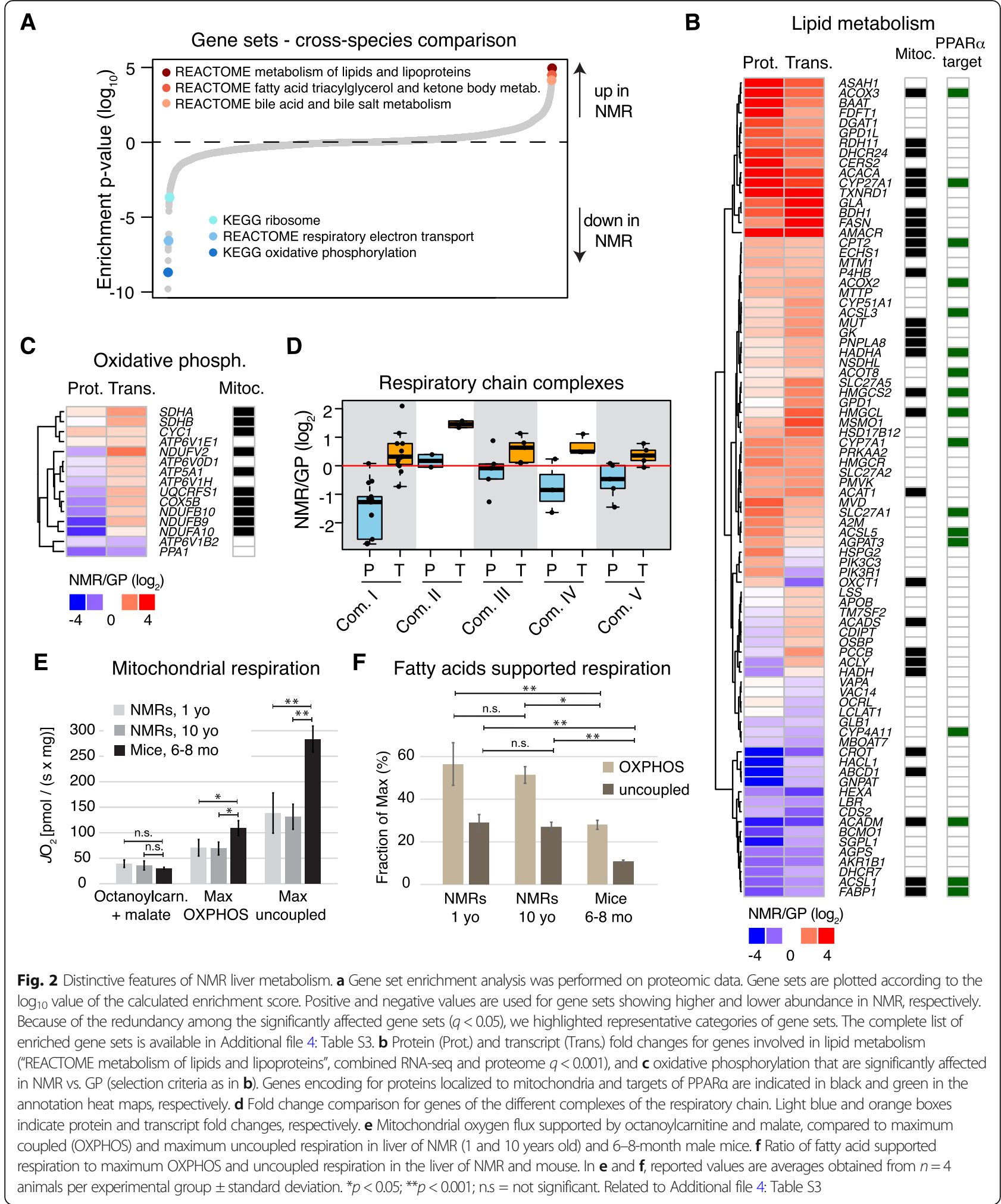

compared them to adult male mice $(6-8$ months, $n=4)$. We first compared the contribution of fatty acids to mitochondrial activity using octanoylcarnitine and malate as substrates. After this, maximum coupled respiration-state (OXPHOS-state) was established by further addition of glutamate and succinate. Finally, we completed the test sequence by adding carbonyl cyanide p-(trifluoromethoxy)-phenylhydrazone (FCCP) in order 
to achieve the maximum uncoupled respiration state. We observed (Fig. 2e) that NMRs and mice showed similar rates of mitochondrial respiration supported by fatty acids. In contrast, NMR livers showed reduced maximum mitochondrial activity compared to mouse liver. In line with the reduced abundance of respiratory chain components, mitochondrial oxygen consumption normalized per wet tissue volume was significantly reduced in both maximum OXPHOS $(35 \%(p=0.011)$ and $37 \%(p=0.014)$ in 1- and 10-year-old NMRs, respectively; one-way ANOVA followed by Bonferroni's $t$ test) and maximum uncoupled states $(51 \%(p<0.001)$ and $54 \%(p<0.001))$. This implies an approximately twofold higher fatty acid supported mitochondrial respiration in NMRs compared to mice $(p<0.001$ for 1 -year-old NMR vs. mouse and $p=0.002$ for 10 -year-old NMR vs. mouse; one-way ANOVA followed by Bonferroni's $t$ test; Fig. 2f). Taken together, our data indicate a marked rearrangement of energy metabolism in the liver of NMR characterized by an enhanced lipid metabolism and globally reduced level of mitochondrial respiration.

\section{Cross-species- and aging-related changes correlate}

Given the existing evidences linking both lipid metabolism and oxidative phosphorylation to lifespan [36, 37], we investigated how these pathways are affected during aging in NMRs. We therefore compared the liver proteome of young (3-4 years old, $n=3$ ) and old (> 18 years old, $n=3)$ NMRs using quantitative mass spectrometry (Additional file 1: Table S1 and Additional file 5: Table S4). Because of the rarity of aged NMR samples, the old NMRs were obtained from a different source (zoo) than the young ones. In order to exclude potential biases due to different origin of the two sample groups, we additionally compared two groups of young ( 1 year old, $n=4)$ and middle-aged ( $>10$ years old, $n=4$ ) NMRs sourced from the same facility (Additional file 1: Table S1 and Additional file 5: Table S4). Histological evaluation of NMR liver tissue derived from the different age groups did not reveal significant inflammatory, fatty, or fibrotic changes, while an increased lipofuscin pigment deposition (a known aging marker) was detectable with aging (Additional file 6: Figure S2A).

Unsupervised hierarchical clustering based on the obtained proteome profiles confirmed an effect of aging on the NMR liver proteome in both dataset (Fig. 3a and Additional file 6: Figure S2B). In addition, protein fold changes positively correlated across the two dataset (Pearson $R=0.23, p<2.2 \times 10^{-16}$, considering all the cross-quantified proteins; Pearson $R=0.50, p=1.0 \times 10^{-11}$, considering proteins significantly affected in both dataset $(q<0.1)$; Additional file 6: Figure S2C). These data indicate that a distinct aging signature is detectable in the liver proteome of NMRs and that some of the changes in protein abundance already manifest in middle-aged (>10 years old) animals.

In order to gain insight into molecular networks affected by aging, we performed Gene Ontology (GO) analysis, based on 5237 protein groups quantified by at least two unique peptides between young and old NMRs (Additional file 5: Table S4). The analysis revealed biological processes that are typically affected by the aging process (Fig. 3b). These include increased inflammation and immune response-related proteins [26], a known signature of aging [38], and accumulation of extracellular matrix proteins [39] (Additional file 7: Table S5). Interestingly, we found a statistically significant overlap between proteins differentially abundant in NMR vs. GP, and proteins whose abundance is affected by NMR aging ( 99 proteins, $p=2 \times 10^{-4}$, Fisher's exact test, Fig. 3c and Additional file 8: Table S6). Additionally, we observed a negative correlation between protein fold changes across species and NMR aging (Pearson $R=-$ $0.38, p=0.0001$; Fig. 3d), resulting in a significant difference between cumulative distributions of NMR vs. GP fold changes for proteins up- or downregulated in NMR aging ( $p=0.002$, Kolmogorov-Smirnov test; Fig. 3e). The directionalities of the differences indicate that proteins with decreasing expression during NMR aging tend to have a higher level in young NMR compared to GP, whereas proteins with increasing expression during NMR aging tend to start from a lower level in young NMR than in GP. Statistical significance for overlap $(p=0.008$, Fisher's exact test), anti-correlation of fold changes (Pearson $R=-0.18, p=1.8 \times 10^{-8}$ ), and difference between cumulative distributions $\left(p=3.1 \times 10^{-12}\right.$, Kolmogorov-Smirnov test) can also be observed from RNA-seq data obtained from the same animals (Additional file 6: Figure S2D:F and Additional file 8: Table S6). In particular, we found among the 30 proteins up in NMR vs. GP and down during NMR aging 13 linked to lipid or fatty acid and 5 to xenobiotic metabolism (Fig. 3f). Our data suggest that this group of proteins is involved in sustaining the longevity of NMRs.

\section{Aging affects similar pathways in both NMR and human liver}

In order to generalize our findings to other species and in particular to human aging, we analyzed the proteome of donor liver samples from 12 individuals aged between 31 and 88 years (Additional file 1: Table S1). In this case, we used formalin-fixed and paraffin-embedded (FFPE) samples due to limited availability of healthy liver samples and quantitatively compared the proteomes using a protocol that we have recently developed [40]. We and others have previously shown that this protocol for FFPE proteomics yields results that are consistent with analysis performed on fresh frozen material [41] and obtained by gene expression analysis [40]. Principal component analysis (PCA) 


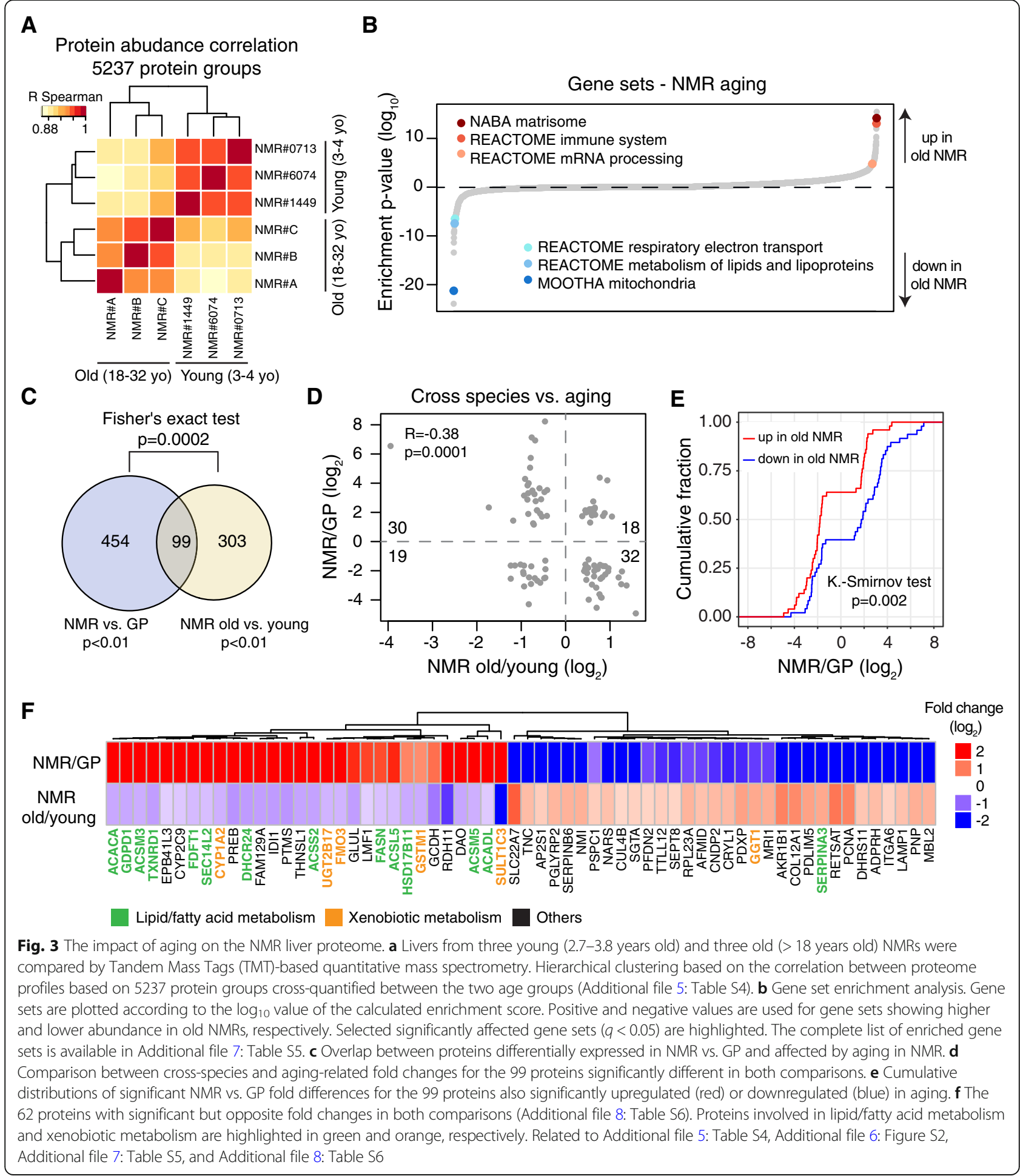

based on 3211 quantified protein groups revealed separation of the proteome profiles based on the age of the donor (Fig. 4a). Guided by the PCA analysis, we split the individuals into two groups defined as adult (below 47 years of age, $n=6$ ) and old (above 66 years, $n=5$ ) and analyzed differential protein expression for 3064 protein groups quantified across all samples (Additional file 9: Table S7). Multiple lines of evidence indicate that aging affects similar pathways in both human and NMR liver. First, as in NMR, GO enrichment analysis revealed an age-dependent decline of proteins involved in lipid metabolism and detoxification of xenobiotics, and an increase of 

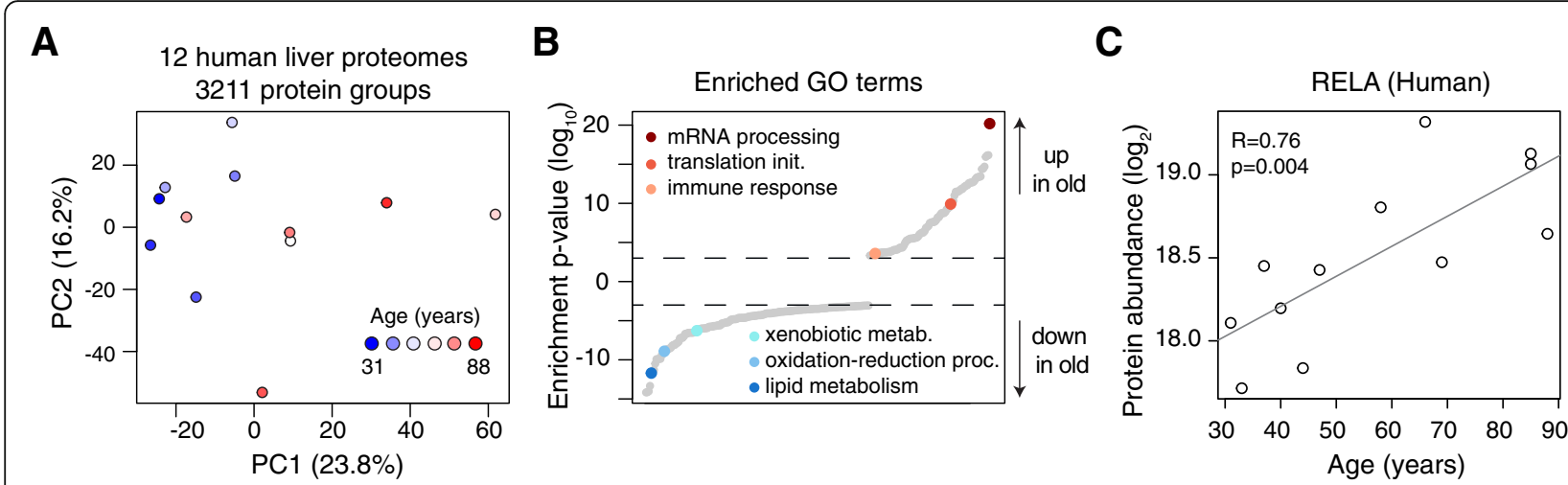

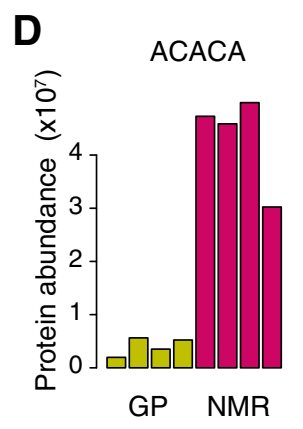

$\mathbf{F}$

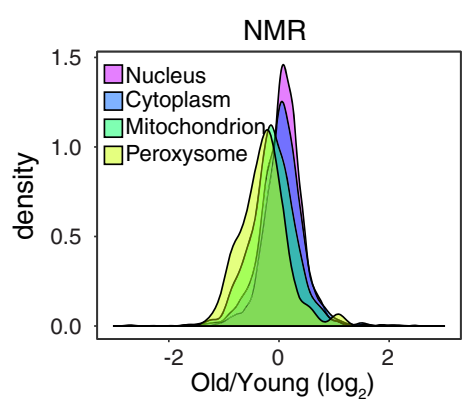

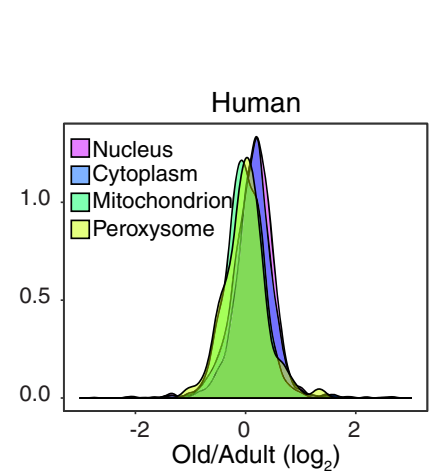

ACACA (Human)

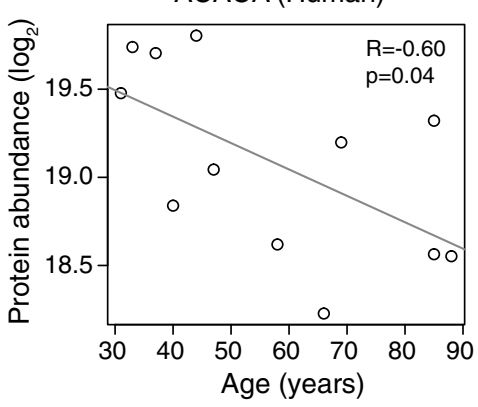

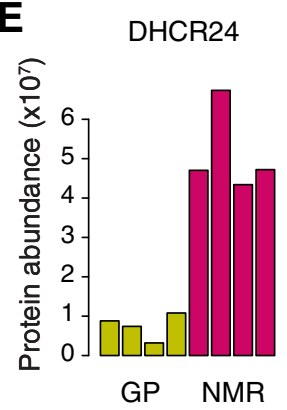

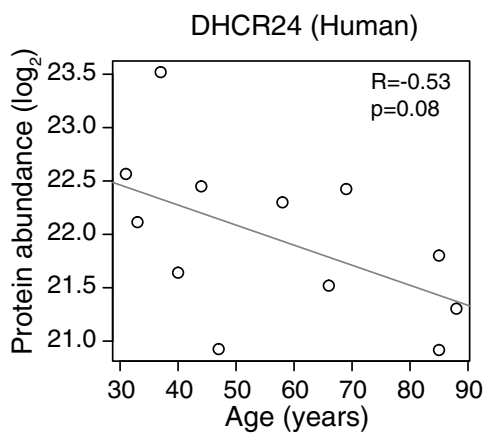

G
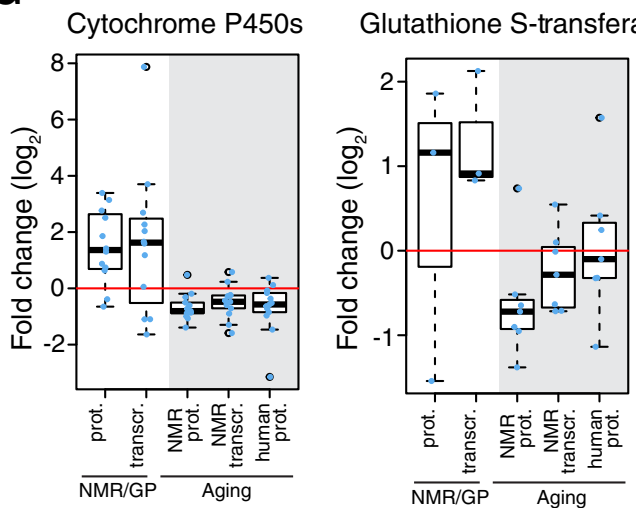

Fig. 4 The impact of aging on the human liver proteome and comparison to NMR aging. a Principal component analysis (PCA) of the human proteome profiles based on 3211 protein groups quantified. b Gene Ontology (GO) enrichment analysis based on differential protein expression between adult ( $<47$ years old, $n=6$ ) and old ( $>66$ years old, $n=5$ ) donors. One donor aged 58 years old was excluded from differential expression analysis. GO categories are plotted according to the $\log _{10}$ value of the calculated enrichment $p$ value. Positive and negative values are used for gene sets showing higher and lower abundance in old individuals, respectively. Selected significantly affected GO terms $(q<0.05)$ are highlighted. The complete list of enriched GO terms is available in Additional file 9: Table S7. c The inflammation marker RELA shows a steady increase of abundance with age. $\mathbf{d}$, e Selected examples of enzymes involved in lipid metabolism being upregulated in NMR vs. GP and decreasing during aging both in NMRs and humans. $\mathbf{f}$ Mitochondrial and peroxisomal proteins decrease with age in both the NMR and human liver. Distributions of fold changes were calculated separately for proteins assigned to different cellular compartments. Mitochondrial $\left(n=953, p=1.2 \times 10^{-37}\right.$ Welch two sample $t$ test for NMR; $n=716, p=1.4 \times 10^{-21}$ Welch two sample $t$ test for human) and peroxisomal proteins ( $n=95, p=4.9 \times 10^{-6}$ Welch two sample $t$ test for NMR; $n=74$, $p=8.9 \times 10^{-3}$ Welch two sample $t$ test for human) showed a significant lower abundance in old samples as compared to the rest of the quantified proteins. $\mathbf{g}$ Major categories of detoxifying enzymes differentially expressed in NMR vs. GP and affected by aging in both NMR and humans (only significantly affected cases are shown for each group; cutoffs: NMR vs. GP and NMR aging, combined $q<0.05$; human proteome aging $q<0.1$ ). Related to Additional file 9: Table S7 and Additional file 10: Figure S3

proteins related to immune response (Fig. $4 \mathrm{~b}$ and Additional file 9: Table S7), and inflammation markers such as RELA/p65 (Fig. 4c). Second, enzymes involved in lipid synthesis such as ACACA and DHCR24, which were found to be expressed at higher level in NMR vs. GP and to decline during NMR aging, showed a negative correlation with the age of the donor (Fig. 4d, e). Enzymes involved in fatty acid beta-oxidation, including ACAA2 and 
HADHA, also showed a trend of lower abundance in the livers from older individuals (Additional file 10: Figure S3A). Third, these changes in metabolic enzymes underline a more general reorganization of the liver proteome that is characterized by a significant reduction of both mitochondrial and peroxisomal proteins during aging in both NMR and human (Fig. 4f). Fourth, multiple proteins involved in different steps of the xenobiotic metabolism showed similar trends. Cytochrome P450s, a subset of Glutathione S-transferases (GSTs), and UDP-glucuronosyltransferases (UGTs) were in most cases expressed at higher levels in NMR vs. GP and showed an age-dependent decline both in NMR and in human (Fig. 4g and Additional file 10: Figure S3B). Taken together, these data indicate that conserved pathways linked to lipid metabolism and detoxification of xenobiotics are affected in both NMR and humans during aging.

The detoxifying enzyme SULT1C3 limits lifespan in C. elegans In order to demonstrate a functional link between these pathways and longevity, we focused on proteins involved in xenobiotic metabolism and tested their ability to influence lifespan in the nematode C. elegans. We chose the phase-II enzyme SULT1C3 and its paralog SULT1C2, since these enzymes are among the most prominently expressed in NMR vs. GP, and they progressively decline during aging starting already at 10 years of age (Fig. 5a, Additional file 6: Figure S2B and Additional file 11: Figure S4). The C. elegans ortholog of SULTIC2/3 is SSU-1 (coded by $s s u-1$ ), and it was previously shown to be expressed at high levels in long-lived and stress-resistant dauer larvae (surviving up to 4 months compared to 2-3-week lifespan of a reproducing adult). High expression levels of ssu-1 are thus associated with extended longevity also in worms [42]. The feeding of wild-type worms with RNAi against ssu-1 from L4 larval stage on resulted in significant reduction of their lifespan (median lifespan reduction of 13\%) suggesting that ssu-1 is required for normal lifespan of nematodes (Fig. 5b). The remarkable stress resistance of dauer larvae is largely due to enhanced expression of pro-survival and detoxification genes mediated by DAF-16/FOXO transcription factor [43, 44]. To test whether the FOXO pathway regulates the expression of ssu-1 during adulthood and aging, we performed an epistasis experiment treating daf-16-deficient nematodes with ssu-1 RNAi. The lifespan-reducing effect of the ssu-1 RNAi is also observed in daf-16 mutants (median lifespan reduction of 12\%) (Fig. 5c), suggesting that the function of ssu-1 in normal aging is regulated by factors other than DAF-16/FOXO. Taken together, these data show that detoxifying enzymes highly expressed in NMR can limit lifespan in a distantly related species.

\section{Discussion}

Cross-species comparisons based on transcriptional profiling have highlighted pathways that correlate with lifespan [1]. In this work, we decided to concentrate on the NMR, as an outlier of exceptional longevity, and directly relate the identified proteome changes to the ones observed in humans, another of those outliers. In our NMR vs. GP comparative approach, we generally observed an expected correlation between transcriptome and proteome differences, confirming that a great fraction of adaptation to local ecosystems occurs via changes in gene expression

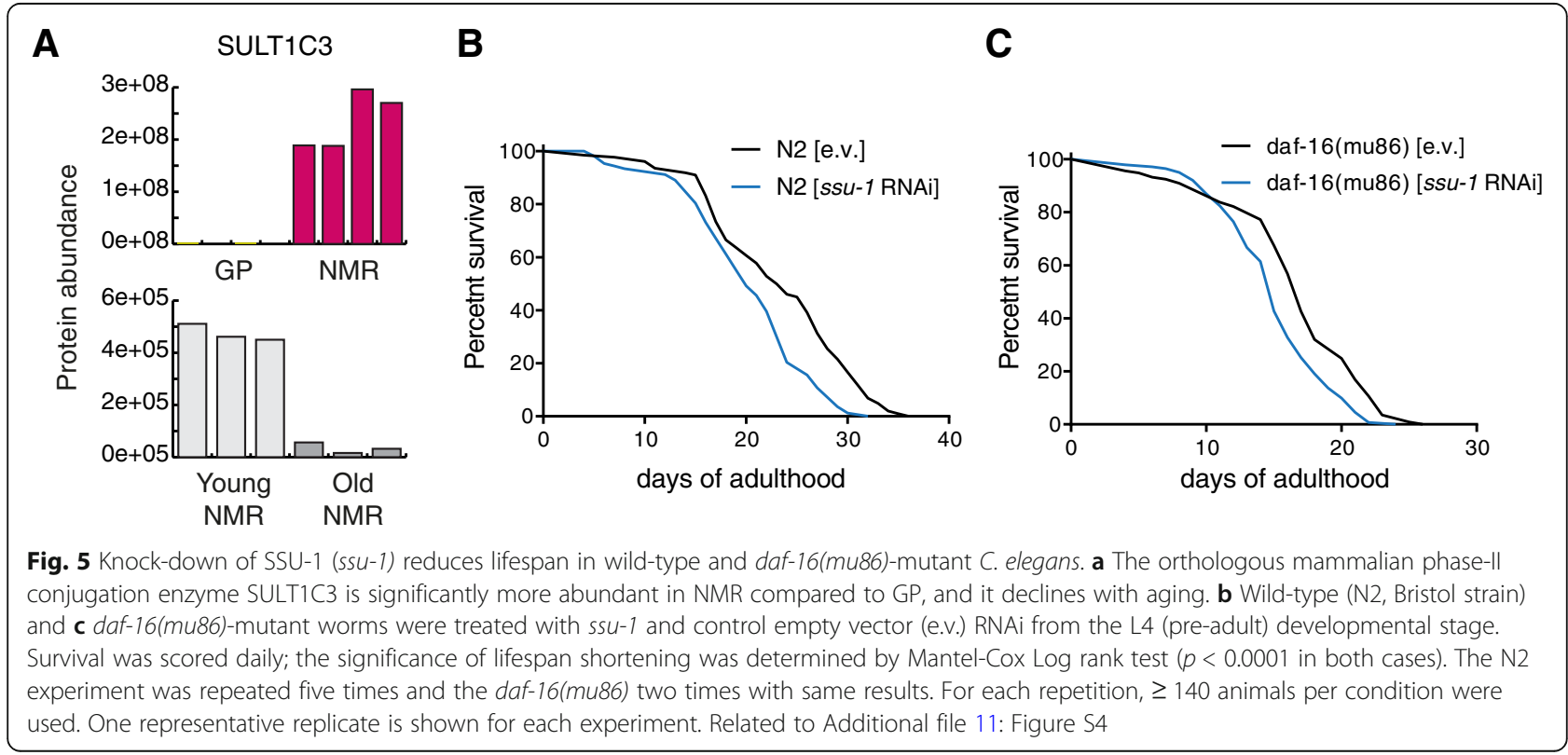


that translate into abundance changes of proteins. However, we have identified changes, particularly among complexes of the mitochondrial respiratory chain, which manifested exclusively at the proteome level. Importantly some of these changes are inline with measureable difference in mitochondrial activity in NMR.

Our cross-species analysis revealed that the liver of NMRs possesses three major characteristics compared to GP: (i) lower rate of mitochondrial respiration, due to reduced protein levels of complex I; (ii) higher reliance on fatty acids for energy production, deriving from increased abundance of enzymes responsible for lipid turnover; and (iii) increased expression of detoxifying enzymes. Importantly, previous works identified GO categories and pathways related to mitochondrion and fatty acid metabolism [8], as well as genes involved in the catabolism of aromatic and organic cyclic compounds [45], to be differentially expressed also in NMRs vs. mice, supporting the peculiarity of NMR liver metabolism among rodents. Furthermore, a transcriptome comparison of livers from the mole-rat Spalax and Rattus norvegicus found enrichment of GO terms related to lipid and fatty acid metabolism among genes expressed at higher levels in Spalax [46], indicating that similar metabolic adaptations might support longevity also in other mole-rat species.

Naked mole-rats have a very low metabolic rate, which reaches only $40 \%$ of the value predicted for a mammal in relation to body mass [47]. They further have a very poor ability of thermoregulation and one of the lowest body temperatures of $32{ }^{\circ} \mathrm{C}$ known among mammals, which has been recorded in captivity [48] and in the wild [49]. These traits likely serve as energy-saving adaptations to their arid environment. Keil et al. reported an inverse relationship between body temperature and expected lifespan, which suggests a contribution of the low body temperature of NMR to their longevity [50]. These adaptations, as well as their high resistance to hypoxia [51], may account for a large proportion of the unique metabolic differences of NMR compared to other mammals.

Consistent with the established and published knowledge on NMR phenotypes at old age, we have shown a clear impact of aging on the NMR liver proteome that negatively affects the abundance of proteins involved in lipid metabolism and detoxification processes. The same pathways are similarly affected with aging also in mice [52] and humans. Our observations support the notion of an extremely low, but detectable, rate of aging in NMRs [53].

Two major questions arise from our work: how NMRs have evolved their particular liver metabolism, and how does this contribute to the extreme longevity of these animals? Multiple studies have previously linked the composition of the mitochondrial respiratory chain to lifespan extension in multiple species [37]: altered composition of the respiratory chain has been shown to induce a hormetic response that can extend lifespan in C. elegans [54]; mild inhibition of complex I leads to increased lifespan in the short-lived fish Nothobranchius furzeri [55]; low abundance of the matrix arm of complex I predicts longevity in mice [56]; fibroblasts isolated from long-lived human individuals including centenarians show altered mitochondrial activity with lower complex I-driven ATP synthesis [57].

Similarly, lipid homeostasis and signaling has been linked to health and longevity [36], and changes in lipid metabolism have been shown to mediate the positive effects of anti-aging dietary interventions. Dietary interventions such as calorie restriction (CR) or fasting influence lipid metabolism $[58,59]$. Transcriptomic and metabolomic measurements showed that CR promotes fatty acid fueling of mitochondria in the liver of mice and it is accompanied by changes in body fat composition [60]. Both fatty acid oxidation and lipid metabolism pathways as well as xenobiotic metabolism are induced by $\mathrm{CR}$ in the mouse liver via epigenetic reprogramming [61]. Changes in lipid metabolism are mechanistically linked to activation of stress response pathways that mediate enhanced proteostasis [62], and fatty acid oxidation and functional peroxisomes are required to maintain mitochondria network homeostasis and promote longevity in C. elegans [63]. In humans, shifts in body composition accompany aging including a decrease of lean mass and accumulation of body fat. Fatty acid oxidation by respiring tissue decreases with age in humans [64, 65]; however, there are discordant reports [66]. A decrease in fatty acid oxidation during aging can lead to adipose tissue accumulation and thus contribute to increased systemic inflammation, a major risk factor for aging-associated disease such as type II diabetes [67]. Our data show that in both NMR and human liver, there is a progressive decline of enzymes responsible for fatty acid turnover. These alterations might contribute to changes in energy metabolism that favor the accumulation of adipose tissue and increased inflammation at older age.

From a mechanistic point of view, it is conceivable that adaptation to the particular ecosystem of NMRs has selected for characteristics of energy metabolism that in turn enabled extreme longevity via activation of stress pathways (Fig. 6). Among these, the NFE2L2 pathway, which controls the expression of many of the detoxifying enzymes that we found increased in NMR vs. GP, was shown to have enhanced activity in NMR [13]. The activities of the same pathways tend to decline during aging, as shown here by the decline of their target genes in both NMR and humans and in different model organisms $[68,69]$. It is therefore tempting to speculate that their higher basal activity in the NMR might contribute to its enhanced stress resistance and ultimately delay the 


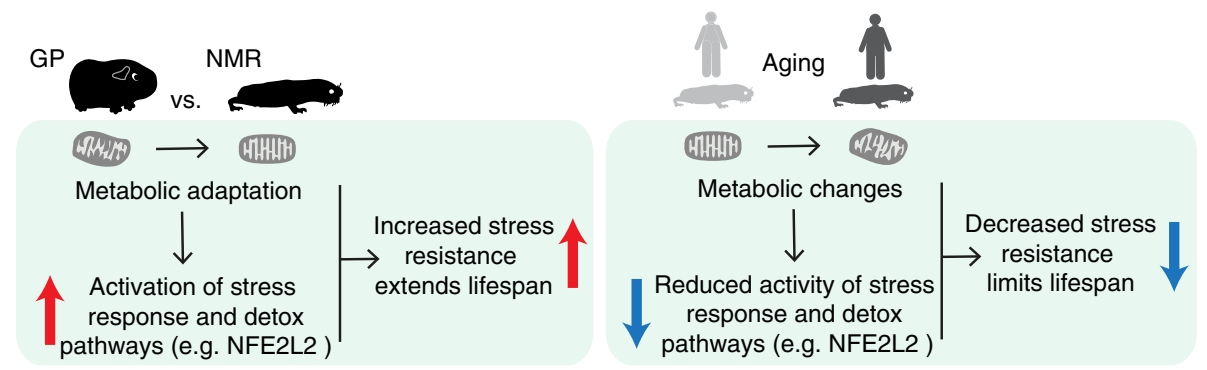

Fig. 6 Proposed model linking metabolic changes to stress response/detoxifying pathways in NMR and during aging

aging process. In line with this hypothesis, genes encoding for respiratory electron transport chain and response to oxidative stress have been shown to be under positive selection in the NMR [7]. Additionally, related molecular networks (lipid metabolism and oxidative stress pathways) are involved in the social status transition from worker to breeder in NMR [34]. This indicates the existence of common evolutionary constrains and molecular mechanisms underlying longevity and eusociality, i.e., reproductive animals' lifelong fecundity coupled with extraordinary life- and healthspan in the NMR [70] and even extended lifespan in other mole-rats [71, 72] and longevity. Further work is required to elucidate in detail which aspects of liver metabolism are sufficient to promote lifespan, and what is the molecular basis mediating positive systemic effects that support organism health by delaying aging in NMR.

\section{Methods}

\section{Ethics approval and consent to participate}

For NMR and GP samples, the sampling and animal procedures were approved by the local ethics committee of the "Landesamt für Gesundheit und Soziales", Berlin, Germany (reference numbers \#ZH 156, G02217/12, and $\mathrm{T} 0073 / 15)$, and were compliant with the national and institutional animal care guidelines. Human liver tissue samples were provided by the tissue bank of the National Center for Tumor Diseases (NCT, Heidelberg, Germany) in accordance with the regulations of the tissue bank and the approval of the ethics committee of Heidelberg University.

\section{Samples}

Young and middle-aged NMR and GP liver tissue samples were obtained at the Leibniz Institute for Zoo and Wildlife Research, IZW (Berlin, Germany). Old NMR liver tissue samples were obtained from the Stockholm Zoo Skansen. All the animals were sacrificed and did not die of natural causes. NMRs from the Stockholm zoo were euthanized as part of veterinary management by the zoo. NMRs were kept inside artificial burrow systems, consisting of acrylic glass containers interconnected with tubes. Except during cleaning and management procedures, animals were kept in complete darkness and supplied daily ad libitum with fresh vegetables. Temperature and humidity were kept stable at $27.0 \pm 2.0{ }^{\circ} \mathrm{C}$ and $85.0 \pm 5.0 \%$, respectively. GPs (breed: Dunkin Hartley HsdDhl:DH, Harlan Laboratories, AN Venray, Netherlands) were kept pairwise in standardized GP cages with a 12-h light-dark cycle. They were fed commercial pellets and fresh vegetables daily; hay and vitamin $\mathrm{C}$-enriched water were provided ad libitum. Temperature and humidity ranged between $18.0 \pm 2.0{ }^{\circ} \mathrm{C}$ and $45.0 \pm 5.0 \%$, respectively. For tissue collection, animals were euthanized by surgical decapitation under general anesthesia (Isofluran CP, CP-Pharma, Burgdorf, Germany). Tissue samples were fresh frozen and stored in liquid nitrogen before transcriptome or proteome analysis.

Human liver samples were obtained in a transplant setting to check the quality of the donor liver by histology before implantation. The age of the donors ranges from 31 to 88 . The tissue samples were formalin fixed, paraffin embedded (FFPE), and slides were stained with hematoxylin and eosin (H\&E). These full-section H\&E slides were re-evaluated by a trained pathologist (SS) confirming that each of the samples used for proteomic analyses did not show any overt pathomorphological changes (e.g., necrosis or significant inflammatory or fatty changes).

\section{RNA sequencing analysis \\ Reference transcripts}

Reference transcripts for NMR are based on recently published de novo transcriptome assembly [73]. Reference transcripts for GP were obtained by de novo transcriptome assembly of ten different tissue samples as described in [34], using the human transcriptome as a reference for gene symbol assignment. Both transcript sets were mapped to the corresponding genomes (NMR UCSC hetgla2, GP UCSC cavpor3) in two steps: BLAT was used to identify the locus, and then SPLIGN (v1.39.8) was applied to splice align the transcript sequence within BLAT locus. 


\section{Transcript quantification}

For NMR age comparison, RNA-seq data were aligned to the reference genome utilizing STAR (v2.4.1d) with a maximum mismatch of $6 \%$ and a minimum aligned length of $90 \%$. Reads mapped to multiple loci were discarded. Gene expression was quantified using HTSEQ (v0.6.1p1) based on the aligned reference transcripts. For cross-species comparison (NMR vs. GP), orthologous transcribed regions were determined using PosiGene [74] with parameter "prank $=0$ max_anchor_gaps_hard=100 rs=NMR." RNA-seq data were aligned to the corresponding orthologous transcribed regions in NMR and GP reference transcripts using bowtie2 (2.2.9) with the parameter "-very-sensitive-local." DESeq2 (v1.6.3) was used to identify DEGs after correction of $p$ values using Benjamini-Hochberg (FDR, denoted as " $q$ ") for NMR aging and cross-species comparison.

\section{Sample preparation for mass spectrometry NMR and GP fresh frozen liver samples for label-free cross- species comparison}

Frozen tissue samples of NMR and GP livers (between 20 and $40 \mathrm{mg}$, Additional file 1: Table S1) were collected into Precellys Lysing kit tubes (Keramik-kit 1.4/2.8 mm, $2 \mathrm{~mL}$ (CKM)) containing $200 \mu \mathrm{L}$ of protein solubilization buffer $(80 \mu \mathrm{M}$ Tris $\mathrm{pH}$ 8.0, $80 \mu \mathrm{M}$ DTT and 4\% SDS) and processed directly. Samples were homogenized in the Precellys 24 homogenizer (Bertin Instruments, France) at $5000 \mathrm{rpm}$ for $30 \mathrm{~s}$ at $4{ }^{\circ} \mathrm{C}$. Samples were then spun down and the supernatant transferred to a 1.5-mL Eppendorf tube. Samples were sonicated using a Bioruptor Plus (Diagenode) for $7.5 \mathrm{~min}$ ( 5 cycles, $1 \mathrm{~min}$ on, $30 \mathrm{~s}$ off, $20^{\circ} \mathrm{C}$ ) using the high setting, and then boiled for $10 \mathrm{~min}$ at $95{ }^{\circ} \mathrm{C}$. A second round of sonication (as before) followed the boiling. Samples were spun down at $20800 \times g$ for $5 \mathrm{~min}$ and the lysate supernatant transferred to fresh tubes. Protein concentration was determined by BCA assay (Pierce) using standard protocol and adjusted to $10 \mu \mathrm{g} / \mu \mathrm{L}$ using solubilization buffer. Five microliters of tissue lysate, corresponding to $50 \mu \mathrm{g}$ protein, was taken for preparation for MS. Cysteine residues were alkylated by adding $1 \mu \mathrm{L}$ of $200 \mathrm{mM}$ iodoacetamide to a final concentration of $15 \mathrm{mM}$ (incubated for $30 \mathrm{~min}$ at room temperature in the dark). Reaction was quenched by addition of $1 \mu \mathrm{L}$ of $200 \mathrm{mM}$ DTT. Sample clean-up proceeded following a modified SP3 protocol. Sera-Mag Speed Beads (\#45152105050250 and \#65152105050250, Thermo Scientific) were mixed $1: 1$, rinsed with water, and stored as a $40-\mu \mathrm{g} / \mu \mathrm{L}$ stock solution in $4{ }^{\circ} \mathrm{C}$, as described in [75]. Four microliters of beads stock was added to the reaction tube and mixed by pipetting, then $11 \mu \mathrm{L}$ acetonitrile containing 5\% $(v / v)$ formic acid was added. Samples were incubated for $8 \mathrm{~min}$ at room temperature to allow protein bindings to the beads. Next, tubes were placed on the magnetic rack.
Supernatant was removed and discarded. Beads were washed twice with $180 \mu \mathrm{L}$ of $70 \%(v / v)$ ethanol and once with $180 \mu \mathrm{L}$ of $100 \%$ acetonitrile. After removal of acetonitrile, beads were air-dried for $60 \mathrm{~s}$ and then resuspended in $7 \mu \mathrm{L}$ of digestion buffer $(6 \mu \mathrm{L} 4 \mathrm{M}$ urea in $100 \mathrm{mM}$ ammonium bicarbonate and $1 \mu \mathrm{L}$ of $1 \mu \mathrm{g} / \mu \mathrm{L}$ of LysC (Wako)). Samples were sonicated for $5 \mathrm{~min}$ in water bath, incubated for $5 \mathrm{~min}$ at $37^{\circ} \mathrm{C}$, and then mixed by pipetting. Digestion was allowed to proceed for $4 \mathrm{~h}$ at $37{ }^{\circ} \mathrm{C}$. After the first step of digestion, beads were resuspended by pipetting, urea was diluted to the final concentration of $1.5 \mathrm{M}$, and $1 \mu \mathrm{L}$ of $1 \mu \mathrm{g} / \mu \mathrm{L}$ of sequencing grade trypsin (Promega) was added to samples. Digestion was performed overnight at $37^{\circ} \mathrm{C}$. After digestion, beads were resuspended by pipetting. One hundred percent acetonitrile was added to the final concentration of $95 \%(v / v)$, and samples were incubated for $8 \mathrm{~min}$ at room temperature. Tubes were placed on the magnetic rack and washed twice with $100 \%$ acetonitrile. Supernatant was removed and beads air-dried and reconstituted in $20 \mu \mathrm{L}$ of $2 \%$ DMSO followed by 5 min of sonication in the water bath. Samples were resuspended by pipetting and placed on the magnetic rack. Supernatant containing peptides was transferred to a fresh tube and acidified with $2 \mu \mathrm{L}$ of $1 \%(v / v)$ formic acid prior to pre-fractionation by high $\mathrm{pH}$ reverse phase chromatography. Offline high $\mathrm{pH}$ reverse phase fractionation was performed using an Agilent 1260 Infinity HPLC System equipped with a binary pump, degasser, variable wavelength UV detector (set to 220 and $254 \mathrm{~nm}$ ), peltier-cooled autosampler (set at $10{ }^{\circ} \mathrm{C}$ ), and a fraction collector. The column was a Waters XBridge C18 column $(3.5 \mu \mathrm{m}, 100 \times 1.0 \mathrm{~mm}$, Waters) with a Gemini C18, $4 \times$ $2.0 \mathrm{~mm}$ SecurityGuard (Phenomenex) cartridge as a guard column. The solvent system consisted of $20 \mathrm{mM}$ ammonium formate $(\mathrm{pH} 10.0)$ as mobile phase (A) and 100\% acetonitrile as mobile phase (B). The separation was accomplished at a mobile phase flow rate of $0.1 \mathrm{~mL} / \mathrm{min}$ using a linear gradient from $100 \%$ A to $35 \%$ B in $61 \mathrm{~min}$. Thirty-four fractions were collected along with the LC separation, which were subsequently pooled into 10 fractions. Pooled fractions were dried in a Speed-Vac and then stored at $-80^{\circ} \mathrm{C}$ until LC-MS/MS analysis.

\section{NMR frozen liver samples for TMT-based comparison of young, middle-aged, and old samples}

For each experimental animal (Additional file 1: Table S1), $100 \mu \mathrm{g}$ protein lysate from the bead-beaten stock of tissue described above were taken up to a final volume of $50 \mu \mathrm{L}$ with $100 \mathrm{mM}$ HEPES buffer, $\mathrm{pH}$ 8.5. Five microliters of $2 \%$ SDS was added, prior to biorupting (5 cycles, $1 \mathrm{~min}$ on, $30 \mathrm{~s}$ off, $20{ }^{\circ} \mathrm{C}$ ) at the highest settings. Samples were spun down at $20800 \times g$ for $1 \mathrm{~min}$, and the lysate supernatant transferred to fresh tubes. Reduction was performed with $2.9 \mu \mathrm{L}$ DTT $\left(200 \mathrm{mM}\right.$ ) for $15 \mathrm{~min}$ at $45{ }^{\circ} \mathrm{C}$ 
before alkylation with $200 \mathrm{mM}$ IAA ( $5 \mu \mathrm{L}, 30 \mathrm{~min}$, room temperature, in the dark). Proteins were then precipitated with four volumes of ice cold acetone to one volume sample and left overnight at $-20{ }^{\circ} \mathrm{C}$. The samples were then centrifuged at $20800 \times g$ for $30 \mathrm{~min}, 4{ }^{\circ} \mathrm{C}$. After removal of the supernatant, the precipitates were washed twice with $500 \mu \mathrm{L} 80 \%(v / v)$ acetone (ice cold). After each wash step, the samples were vortexed, then centrifuged again for $2 \mathrm{~min}$ at $4{ }^{\circ} \mathrm{C}$. The pellets were then allowed to air-dry before being dissolved in digestion buffer $(50 \mu \mathrm{L}, 3 \mathrm{M}$ urea in 0, $1 \mathrm{M}$ HEPES, $\mathrm{pH} 8 ; 1 \mu \mathrm{g} \mathrm{LysC}$ ) and incubated for $4 \mathrm{~h}$ at $37{ }^{\circ} \mathrm{C}$ with shaking at $600 \mathrm{rpm}$. Then, the samples were diluted 1:1 with milliQ water (to reach $1.5 \mathrm{M}$ urea) and were incubated with $1 \mu \mathrm{g}$ trypsin for $16 \mathrm{~h}$ at $37^{\circ} \mathrm{C}$. The digests were then acidified with $10 \%$ trifluoroacetic acid and then desalted with Waters Oasis $^{\bullet}$ HLB $\mu$ Elution Plate $30 \mu \mathrm{m}$ in the presence of a slow vacuum. In this process, the columns were conditioned with $3 \times 100 \mu \mathrm{L}$ solvent B (80\% (v/v) acetonitrile; $0.05 \%(v / v)$ formic acid) and equilibrated with $3 \times 100 \mu \mathrm{L}$ solvent A $(0.05 \%(v / v)$ formic acid in milliQ water). The samples were loaded, washed three times with $100 \mu \mathrm{L}$ solvent $\mathrm{A}$, and then eluted into PCR tubes with $50 \mu \mathrm{L}$ solvent B. The eluates were dried down with the speed vacuum centrifuge and dissolved in 200 mM HEPES buffer, $\mathrm{pH} 8.5$ for TMT labeling. Twenty-five micrograms of peptides were taken for each labeling reaction at $1 \mu \mathrm{g} / \mu \mathrm{L}$ concentration. TMT-6plex reagents for old vs. young comparison (TMT-10plex for middle-aged vs. young comparison) (Thermo Scientific) were reconstituted in $41 \mu \mathrm{L} 100 \%$ anhydrous DMSO. TMT labeling was performed by addition of $2.5 \mu \mathrm{L}$ of the TMT reagent. After $30 \mathrm{~min}$ of incubation at room temperature, with shaking at $600 \mathrm{rpm}$ in a thermomixer (Eppendorf), a second portion of TMT reagent $(2.5 \mu \mathrm{L})$ was added and incubated for another $30 \mathrm{~min}$. The reaction was quenched with $1 \mu \mathrm{L}$ of $20 \mathrm{mM}$ lysine in $100 \mathrm{mM}$ ammonium bicarbonate. After checking labeling efficiency, samples were pooled (48 $\mu \mathrm{g}$ total), cleaned once again with Oasis and subjected to high $\mathrm{pH}$ fractionation prior to MS analysis. For middle-aged vs. young comparison, two additional samples were generated by pooling the young and middle-aged samples separately and used to fill the two remaining TMT channels. Offline high $\mathrm{pH}$ reverse phase fractionation was performed as described above with the following modifications for TMT-labeled samples: (i) the separation was accomplished at a mobile phase flow rate of $0.1 \mathrm{~mL} / \mathrm{min}$ using a non-linear gradient from $95 \%$ A to $40 \%$ B in 91 min; (ii) 48 fractions were collected along with the LC separation that were subsequently pooled into 16 fractions.

\section{Human FFPE liver samples}

The specimens were cut on a microtome into 5 - $\mu$ m-thick sections, mounted on glass slides and processed with a modified version of the protocol described in [40]. Slides were deparaffinized in xylene for $2 \times 5 \mathrm{~min}$, rehydrated in $100 \%$ ethanol for $2 \times 5 \mathrm{~min}$, and then washed in $96 \%(\mathrm{v} / \mathrm{v})$, $70 \%(v / v), 50 \%(v / v)$ ethanol and milliQ water for $1 \times 5 \mathrm{~min}$ each. Region of interest were gently scraped using a scalpel and transferred to a PCR tube containing $100 \mu \mathrm{L}$ of protein solubilization buffer $(80 \mu \mathrm{M}$ Tris $\mathrm{pH}$ 8.0, $80 \mu \mathrm{M}$ DTT, and $4 \%$ SDS) and processed directly. Samples were sonicated using a Bioruptor Plus (Diagenode) for $25.2 \mathrm{~min}$ (15 cycles, $1 \mathrm{~min}$ on, $30 \mathrm{~s}$ off) at $20^{\circ} \mathrm{C}$ using the high setting, and then boiled for $1 \mathrm{~h}$ at $99^{\circ} \mathrm{C}$. Sonication followed by boiling was performed twice. Cysteine residues were alkylated by adding $200 \mathrm{mM}$ iodoacetamide to a final concentration of $15 \mathrm{mM}$ (incubated for $30 \mathrm{~min}$ at room temperature in the dark). Reaction was quenched by addition of $10 \mu \mathrm{L}$ of $200 \mathrm{mM}$ DTT. Protein were then acetone precipitated, digested, and desalted as described above for NMR samples (aging comparison), with the exceptions that $0.5 \mu \mathrm{g}$ of both LysC and trypsin were used instead of $1 \mu \mathrm{g}$ to accommodate for the lower amount of protein extract employed, and no TMT labeling was performed.

\section{Mass spectrometry data acquisition Label-free analysis of NMR and GP liver samples}

For label-free experiments, each fraction from the four GP and four NMR samples, separated by high $\mathrm{pH}$, were resuspended in $10 \mu \mathrm{L}$ reconstitution buffer $(5 \%(v / v)$ acetonitrile, $0.1 \%(v / v)$ TFA in water) and $8 \mu \mathrm{L}$ were injected. Peptides were separated using the nanoAcquity UPLC system (Waters) fitted with a trapping (nanoAcquity Symmetry C18, $5 \mu \mathrm{m}, 180 \mu \mathrm{m} \times 20 \mathrm{~mm}$ ) and an analytical column (nanoAcquity $\mathrm{BEH} \mathrm{C} 18,2.5 \mu \mathrm{m}$, $75 \mu \mathrm{m} \times 250 \mathrm{~mm}$ ). The outlet of the analytical column was coupled directly to an Orbitrap Fusion Lumos (Thermo Fisher Scientific) using the Proxeon nanospray source. Solvent A was water, $0.1 \%(v / v)$ formic acid, and solvent B was acetonitrile, $0.1 \%(v / v)$ formic acid. The samples were loaded with a constant flow of solvent A at $5 \mu \mathrm{L} / \mathrm{min}$, onto the trapping column. Trapping time was $6 \mathrm{~min}$. Peptides were eluted via the analytical column at a constant flow of $0.3 \mu \mathrm{L} / \mathrm{min}$, at $40{ }^{\circ} \mathrm{C}$. During the elution step, the percentage of solvent $\mathrm{B}$ increased in a linear fashion from 5 to $7 \%$ in $10 \mathrm{~min}$, then from $7 \% \mathrm{~B}$ to $30 \% \mathrm{~B}$ in a further $105 \mathrm{~min}$ and to $45 \% \mathrm{~B}$ by $130 \mathrm{~min}$. The peptides were introduced into the mass spectrometer via a Pico-Tip Emitter $360 \mu \mathrm{m}$ OD $\times 20 \mu \mathrm{m}$ ID; $10 \mu \mathrm{m}$ tip (New Objective), and a spray voltage of $2.2 \mathrm{kV}$ was applied. The capillary temperature was set at $300{ }^{\circ} \mathrm{C}$. Full-scan MS spectra with mass range $375-1500 \mathrm{~m} / \mathrm{z}$ were acquired in profile mode in the Orbitrap with resolution of 120,000 FWHM using the quad isolation. A first batch of samples (NMR F1-6074, M1-1449; GP \#18, \#19) was acquired with the following settings. The RF on the ion funnel was set to $60 \%$. The filling time was set at maximum of $100 \mathrm{~ms}$ with an AGC target of $4 \times 10^{5}$ ions and 1 
microscan. The peptide monoisotopic precursor selection was enabled along with relaxed restrictions if too few precursors were found. The most intense ions (instrument operated for a $3 \mathrm{~s}$ cycle time) from the full scan MS were selected for MS2, using quadrupole isolation and a window of $1.6 \mathrm{Da}$. An intensity threshold of $5 \times 10^{3}$ ions was applied. Higher-energy collisional dissociation (HCD) was performed with collision energy of $35 \%$. A maximum fill time of 30 ms with an AGC target of $1 \times 10^{4}$ for each precursor ion was set. MS2 data were acquired in centroid in the ion trap, in Rapid scan mode, with fixed first mass of $120 \mathrm{~m} / \mathrm{z}$. The dynamic exclusion list was with a maximum retention period of $60 \mathrm{~s}$ and relative mass window of $10 \mathrm{ppm}$. In order to improve the mass accuracy, internal lock mass correction using a background ion $(\mathrm{m} / \mathrm{z}$ 445.12003) was applied. For data acquisition and processing of the raw data, Xcalibur 4.0 (Thermo Scientific) and Tune version 2.0 were employed. As a consequence of method optimization, the following parameters were changed for a second batch of samples (NMR \#0713, \#4925; GP \#23, \#28): RF on the ion funnel was set to $40 \%$, AGC target to $2 \times 10^{5}$, quadrupole isolation window to $1.4 \mathrm{Da}, \mathrm{HCD}$ collision energy to $30 \%$, fill time to $300 \mathrm{~ms}$, AGC target to $2 \times 10^{3}$, and the instrument was set to inject ions for all available parallelizable time. Since the two batches of samples were block randomized (i.e., both contained the same number of NMR and GP samples), the usage of two different methods did not influence the outcome of our comparison, as shown by the expected clustering of the samples according to the species of origin (Fig. 1a).

\section{TMT analysis of NMR young, middle-aged, and old samples}

For TMT experiments, fractions were resuspended in $10 \mu \mathrm{L}$ reconstitution buffer $(5 \%(v / v)$ acetonitrile, $0.1 \%$ $(v / v)$ TFA in water) and $3.5 \mu \mathrm{L}$ were injected. Peptides were analyzed using the same LC-MS/MS setup described above with the following modifications. Peptides were eluted using a linear gradient from 5 to $7 \%$ in $10 \mathrm{~min}$, then from $7 \% \mathrm{~B}$ to $30 \% \mathrm{~B}$ in a further $105 \mathrm{~min}$ and to $45 \%$ B by $130 \mathrm{~min}$. Full-scan MS spectra with mass range $375-1500 \mathrm{~m} / \mathrm{z}$ were acquired in profile mode in the Orbitrap with resolution of 60,000 FWHM using the quad isolation. The RF on the ion funnel was set to $40 \%$. The filling time was set at maximum of $100 \mathrm{~ms}$ with an AGC target of $4 \times 10^{5}$ ions and 1 microscan. The peptide monoisotopic precursor selection was enabled along with relaxed restrictions if too few precursors were found. The most intense ions (instrument operated for a $3 \mathrm{~s}$ cycle time) from the full scan MS were selected for MS2, using quadrupole isolation and a window of $1 \mathrm{Da}$. HCD was performed with collision energy of $35 \%$. A maximum fill time of $50 \mathrm{~ms}$ for each precursor ion was set. MS2 data were acquired with fixed first mass of $120 \mathrm{~m} / z$. The dynamic exclusion list was with a maximum retention period of $60 \mathrm{~s}$ and relative mass window of $10 \mathrm{ppm}$. For the MS3, the precursor selection window was set to the range $400-2000 \mathrm{~m} / z$, with an exclude width of $18 \mathrm{~m} / z$ (high) and $5 \mathrm{~m} / z$ (low). The most intense fragments from the MS2 experiment were co-isolated (using Synchronus Precursor Selection = 8) and fragmented using HCD (65\%). MS3 spectra were acquired in the Orbitrap over the mass range $100-1000 \mathrm{~m} / \mathrm{z}$ and resolution set to 30,000 FWMH. The maximum injection time was set to $105 \mathrm{~ms}$, and the instrument was set not to inject ions for all available parallelizable time.

\section{Data-independent acquisition (DIA) for human FFPE samples} Peptides were spiked with retention time HRM kit (Biognosys AG) and analyzed using the same LC-MS/ MS setup described above with the following modifications. Approx. $1 \mu \mathrm{g}$ for data-dependent acquisition (DDA) and $3 \mu \mathrm{g}$ for DIA analysis were loaded. Peptides were eluted via a non-linear gradient from 0 to $40 \%$ in $120 \mathrm{~min}$. Total runtime was $145 \mathrm{~min}$, including clean-up and column re-equilibration. The RF lens was set to $30 \%$. For spectral library generation, a pooled sample was generated by mixing equal portion of each sample, injected six times, and measured in DDA mode. The conditions for DDA data acquisition were as follows: Full-scan MS spectra with mass range 350-1650 m/z were acquired in profile mode in the Orbitrap with resolution of 60,000 FWHM. The filling time was set at maximum of $50 \mathrm{~ms}$ with limitation of $2 \times 10^{5}$ ions. The "Top Speed" method was employed to take the maximum number of precursor ions (with an intensity threshold of $5 \times 10^{4}$ ) from the full-scan MS for fragmentation (using HCD collision energy, 30\%) and quadrupole isolation (1.4 $\mathrm{Da}$ window) and measurement in the Orbitrap (resolution 15,000 FWHM, fixed first mass $120 \mathrm{~m} / z$ ), with a cycle time of $3 \mathrm{~s}$. The MIPS (monoisotopic precursor selection) peptide algorithm was employed but with relaxed restrictions when too few precursors meeting the criteria were found. The fragmentation was performed after accumulation of $2 \times 10^{5}$ ions or after filling time of $22 \mathrm{~ms}$ for each precursor ion (whichever occurred first). MS/MS data were acquired in centroid mode. Only multiply charged $\left(2^{+}-7^{+}\right)$precursor ions were selected for MS/MS . Dynamic exclusion was employed with maximum retention period of $15 \mathrm{~s}$ and relative mass window of $10 \mathrm{ppm}$. Isotopes were excluded. For data acquisition and processing, Tune version 2.1 was employed.

For the DIA data acquisition, the same gradient conditions were applied to the LC as for the DDA and the MS conditions were varied as follows: Full-scan MS spectra with mass range $350-1650 \mathrm{~m} / \mathrm{z}$ were acquired in profile mode in the Orbitrap with resolution of 120,000 FWHM. The filling time was set at maximum of $20 \mathrm{~ms}$ with limitation of $5 \times 10^{5}$ ions. DIA scans were acquired 
with 34 mass window segments of differing widths across the MS1 mass range with a cycle time of $3 \mathrm{~s}$. HCD fragmentation (30\% collision energy) was applied, and MS/MS spectra were acquired in the Orbitrap with a resolution of 30,000 FWHM over the mass range $200-2000 \mathrm{~m} / z$ after accumulation of $2 \times 10^{5}$ ions or after filling time of $70 \mathrm{~ms}$ (whichever occurred first). Ions were injected for all available parallelizable time. Data were acquired in profile mode.

\section{Mass spectrometry data analysis \\ Label-free cross-species comparison of NMR and GP liver samples}

The Andromeda search engine [76], part of MaxQuant (version 1.5.3.28) [77], was used to search the data. The data for GP and NMR were searched separately against translated species-specific reference transcripts (see the "RNA sequencing analysis" section). Databases with a list of common contaminants were appended in both cases. The data were searched with the following modifications: carbamidomethyl (C) (fixed), and oxidation (M) and acetyl (protein $\mathrm{N}$-term) (variable). The mass error tolerance for the full-scan MS spectra was set at $20 \mathrm{ppm}$ and for the MS/MS spectra at $0.5 \mathrm{Da}$. A maximum of two missed cleavages were allowed. Peptide and protein level 1\% FDR were applied using a target-decoy strategy [78]. iBAQ (label-free quantification) values from the MaxQuant output were used to perform cross-species differential protein expression analysis using scripts written in R (v3.4.1). After removal of reverse and contaminant hits, only protein groups quantified by at least two unique peptides were retained. Common human gene symbols were used to combine iBAQ values for NMR and GP samples. Only protein groups quantified in at least two animals per group were retained when comparing protein abundances between NMR and GP. To reduce technical variation, data were $\log _{2}$ transformed and quantile-normalized using the preprocessCore library. Protein differential expression was evaluated using the limma package [79]. Differences in protein abundances were statistically determined using the Student's $t$ test moderated by the empirical Bayes method. $P$ values were adjusted for multiple testing using the Benjamini-Hochberg method (FDR, denoted as " $q$ ") [80] (Additional file 2: Table S2).

\section{TMT-based analysis of young, middle-aged, and old NMR livers}

TMT data were processed using Proteome Discoverer v2.0 (Thermo Fisher Scientific). Data were searched against the NMR fasta database using Mascot v2.5.1 (Matrix Science) with the following settings: Enzyme was set to trypsin, with up to one missed cleavage. MS1 mass tolerance was set to $10 \mathrm{ppm}$ and MS2 to $0.5 \mathrm{Da}$. Carbamidomethyl cysteine was set as a fixed modification and oxidation of methionine as variable. Other modifications included the TMT-6plex modification from the quan method used. The quan method was set for reporter ions quantification with HCD and MS3 (mass tolerance, $20 \mathrm{ppm}$ ). The false discovery rate for peptide-spectrum matches (PSMs) was set to 0.01 using Percolator [81]. Reporter ion intensity values for the filtered PSMs were exported and processed using in-house written $\mathrm{R}$ scripts to remove common contaminants and decoy hits Additionally, only PSMs having reporter ion intensities above $1 \times 10^{3}$ in all the relevant TMT channels were retained for quantitative analysis. Only protein groups quantified by at least two unique peptides were analyzed for differential expression. Data were analyzed using the MSnbase package [82]. Reporter ion intensities were $\log _{2}$-transformed and normalized using the vsn package [83]. Peptide-level data were summarized into their respective protein groups by taking the median value. Differential protein expression was assessed using the limma package, as described above (Additional file 5: Table S4).

\section{DIA analysis of FFPE human samples}

For library creation, the DDA data was searched using the Andromeda search engine built in MaxQuant (version 1.5.3.28). The data were searched against a human database (Swiss-Prot entries of the Uniprot KB database release 2016_01, 20198 entries) with a list of common contaminants appended, as well as the HRM peptide sequences. The data were searched with the following modifications: carbamidomethyl (C) (fixed) and oxidation $(\mathrm{M}) /$ acetyl (protein $\mathrm{N}$-term) (variable). The mass error tolerance for the full-scan MS and MS/MS spectra was set at $20 \mathrm{ppm}$. A maximum of one missed cleavage was allowed. The identifications were filtered to satisfy FDR of $1 \%$ on peptide and protein level. A spectral library was created from the MaxQuant output of the DDA runs combined using Spectronaut (version 10, Biognosys AG). This library contained 34,014 precursors, corresponding to 3295 protein groups using Spectronaut protein inference. DIA data were then uploaded and searched against this spectral library. Precursor matching, protein inference, and quantification were performed in Spectronaut using default settings [84]. Differential protein expression was evaluated using a pairwise $t$ test performed at the precursor level followed by multiple testing correction according to [85]. The data (candidate table, Additional file 9: Table S7) was exported from Spectronaut and used for further data analyses (see below).

\section{Data analysis}

For integrated analysis of RNA-seq and proteomic data, transcripts and protein groups were matched using the corresponding gene symbol, $p$ values were combined 
using the Fisher method, followed by correction for multiple testing using the Benjamini-Hochberg method (FDR, denoted as " $q$ ") [80]. Gene set enrichments (Figs. 2a and $3 \mathrm{~b}$ ) were performed with the $\mathrm{R}$ package gage [86] using gene set definitions from the Molecular Signatures Database (MSigDB, C2 v5.1) [87]. Gene Ontology enrichment analysis (Fig. 4b) was performed using GOrilla [88] giving as input the list of quantified proteins that were ranked according to the level of differential expression (fold change).

\section{Measurements of mitochondrial activity}

Mitochondrial respiration was measured in homogenized liver tissue samples of NMRs and mice by means of high-resolution respirometry using the Oroboros ${ }^{\circ}$ Oxygraph-2K (Oroboros Instruments, Innsbruck, Austria). This device allows for simultaneous recording of the $\mathrm{O}_{2}$ concentration in two parallel chambers calibrated for $2 \mathrm{~mL}$ of respiration medium containing $110 \mathrm{mM}$ D-Sucrose (Sigma 84097), $60 \mathrm{mM}$ K-lactobionate (Aldrich 153516), $0.5 \mathrm{mM}$ ethylene glycol tetra acetic acid (Sigma E4378), $1 \mathrm{~g} / \mathrm{L}$ bovine serum albumin free from essentially fatty acids (Sigma A 6003), 3 mM MgCl 2 (Scharlau MA0036), 20 mM

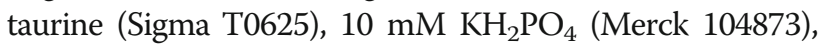
20 mM HEPES (Sigma H7523), adjusted to pH 7.1 with $\mathrm{KOH}$ and equilibrated with $21 \% \mathrm{O}_{2}$ at $37^{\circ} \mathrm{C}$. Mitochondrial respiration was quantified in terms of oxygen flux $\left(\mathrm{JO}_{2}\right)$ calculated as the rate of change of the $\mathrm{O}_{2}$ concentration in the chambers normalized for wet tissue volume.

The liver tissue homogenates were generated from 40 to $50 \mathrm{mg}$ of wet tissue samples suspended in $2 \mathrm{~mL}$ of ice-cold respiration medium. Aliquots of the homogenates were added to each oxygraph chamber in order to obtain a final amount of $4 \mathrm{mg}$ of NMR liver tissue or $2 \mathrm{mg}$ of mouse liver tissue per chamber. The different amount of tissue was chosen in order to obtain similar absolute $\mathrm{JO} 2$ values, i.e., $\mathrm{JO} 2$ values not normalized per wet weight, in both species. Every sample was measured in duplicates; the mean values from both chambers were used for statistical analysis.

The titration sequence used for the experiments was as follows: $2 \mathrm{mM}$ malate $+1 \mathrm{mM}$ octanoylcarnitine, $5 \mathrm{mM}$ ADP, $10 \mu \mathrm{M}$ cytochrome c, $10 \mathrm{mM}$ glutamate, $10 \mathrm{mM}$ succinate, $2.5 \mu \mathrm{M}$ oligomycin, $1 \mu \mathrm{M}$ carbonyl cyanide $\mathrm{p}$-(trifluoromethoxy)-phenylhydrazone (FCCP), $0.5 \mu \mathrm{M}$ rotenone, and $5 \mu \mathrm{M}$ antimycin $\mathrm{A}$. The $\mathrm{JO}_{2}$ values after addition of octanoyl carnitine, malate, ADP, and cytochrome $\mathrm{c}$ allow quantifying fatty acid oxidation. The addition of cytochrome $\mathrm{c}$ after ADP is required to test the integrity of the outer mitochondrial membrane. If homogenization steps damaged the mitochondrial membrane, addition of cytochrome $\mathrm{c}$ induces an increase of the respiratory values. The maximum oxidative capacity of the mitochondrial respiratory chain in the coupled state (maximum OxPhos) was then determined after the subsequent addition of glutamate and succinate. Further injections of the ATP synthase inhibitor oligomycin and of the uncoupler FCCP allowed obtaining the maximum respiratory activity in the uncoupled state. In the next two steps, complex I and complex III were sequentially inhibited by administration of rotenone and antimycin A respectively. Finally, sequentially injecting $2 \mathrm{mM}$ ascorbate and $0.5 \mathrm{mM}$ of the complex IV substrate tetramethyl phenylene diamine (TMPD) in the parallel chambers allowed for selectively quantifying the activity of the cytochrome-c-oxidase (COX). Part of the $\mathrm{JO}_{2}$ induced by the injection of TMPD is caused by auto-oxidation of this compound. Therefore, inhibiting the COX by $40 \mu \mathrm{M}$ sodium sulfide allowed to quantify and thus to subtract this auto-oxidation-related part from the total $\mathrm{JO}_{2}$ value under TMPD.

\section{C. elegans lifespan measurements}

HT115 bacteria containing specific RNAi constructs were grown on lysogeny broth agar plates supplemented with ampicillin and tetracycline. Plates were kept at $4{ }^{\circ} \mathrm{C}$. Overnight cultures were grown in lysogeny broth media containing ampicillin. RNAi expression was induced by adding $1 \mathrm{mM}$ isopropylthiogalactoside (IPTG) and incubating the cultures at $37{ }^{\circ} \mathrm{C}$ for $20 \mathrm{~min}$ before seeding bacteria on NGM agar supplemented with ampicillin and 3 mM IPTG. Synchronized L4 larvae of N2, Bristol strain, and daf-16(mu86) strain were placed on $60-\mathrm{mm}$ dishes containing RNAi expressing bacteria at a density of 70 worms per plate; two replica plates were used for each condition providing a sum of 140 total animals per condition at the start of the test. Worms were transferred to new plates on a daily basis until adulthood day 6 (AD6) and later transferred to new plates every 3-4 days. The number of dead animals was scored daily. Worms were kept at $20{ }^{\circ} \mathrm{C}$ from embryonic stage on and until the end of the experiment. The analysis of the lifespan data including statistics was performed using GraphPad Prism software.

\section{Conclusions}

Using an integrated proteomic and transcriptomic approach, we have revealed differences in the liver proteome of the long-lived NMRs compared to the shorter-lived GPs. We have confirmed a preferential way of utilizing fatty acids to fuel respiration in NMRs, reflecting a distinct composition of their mitochondria. Furthermore, we have identified a progressive signature of aging that manifest in the liver of NMRs on a molecular level. Interestingly, functionally related groups of proteins, including enzymes of the detoxification pathways, were similarly affected by aging in both NMR and human liver samples. This underlines a direct link between the aging processes of these two species. It remains to be demonstrated whether the 
aging-affected pathways influence the health status of old NMRs and limit their lifespan, as we have shown to be the case in the nematode C. elegans.

\section{Additional files}

Additional file 1: Table S1. List of used animals. (XLSX $36 \mathrm{~kb}$ ) Additional file 2: Table S2. NMR vs. GP dataset, contains differential expression analysis for proteomic and transcriptomic data and integrated RNAseq proteome analysis of NMR and GP livers. (XLS $4142 \mathrm{~kb}$ )

Additional file 3: Figure S1. (A) Heatmap showing genes that displayed discordant NMR vs. GP fold changes at the transcript and protein level. Only cases significant in both comparisons $(q<0.1)$ are shown. (B and C) Comparison of transcript levels of PRDX1 and TXNRD1 across multiple tissues. For both genes, transcript levels are increased in NMR vs. GP in the liver $\left(q<2.2 \times 10^{-300}\right.$ for PRDX1; $q=1.5 \times 10^{-45}$ for TXNRD1), while they show similar abundances in the other tissues examined. RNAseq data for heart, skin, kidney and cerebellum were obtained from [34]. Related to Fig. 1 and Additional file 1: Table S1 and Additional file 2: Table S2. (PDF 488 kb)

Additional file 4: Table S3. Excel workbook (.xIsx), NMR vs. GP gene set enrichment, contains significantly affected gene sets from the proteomic analysis. (XLSX $65 \mathrm{~kb}$ )

Additional file 5: Table S4. Excel workbook (.xlsx), NMR aging dataset, contains differential expression analysis for proteomic and transcriptomic data and integrated RNAseq proteome analysis of old vs. young NMR livers, and proteomic analysis of middle-aged vs. young NMR livers. (XLS 6040 kb)

Additional file 6: Figure S2. Comparison of liver proteomes between middle-aged and young NMRs, and correlation analysis of transcript level differences in NMR vs. GP and NMR aging. (A) Representative micrographs show H\&E stained (FFPE) liver tissue sections from NMRs of the indicated age groups. Scale bar $=100 \mu \mathrm{m}$. (B) Livers from 4 young (1 yo) and 4 middle-aged (> 10 yo) NMRs were compared by Tandem Mass Tags (TMT) based quantitative mass spectrometry. Hierarchical clustering based on the correlation between proteome profiles based on 4936 protein groups cross-quantified between the two age groups (Additional file 5: Table S4). (C) Comparison of protein fold changes calculated between old vs. young ( $x$-axis) and middle-aged vs. young ( $y$-axis) NMRs. Colored dots indicate proteins significant $(q<0.1)$ in both comparisons. The names of selected proteins that show consisted fold changes in the two comparisons are indicated. (D) Significant overlap between differentially expressed genes (DEGs) in NMR vs. GP and in aging of NMR. (E) Comparison between crossspecies and aging-related fold changes for the 875 DEGs significant in both comparisons $(q<0.01)$ shows significant negative correlation. (F) Cumulative distributions of NMR vs. GP fold changes for the 875 DEGs also significantly up- (red) or down- (blue) regulated in NMR aging. The $x$-axis was restricted to \pm 8 for display purpose. Related to Fig. 3, Additional file 5: Table S4 and Additional file 8: Table S6. (PDF $13567 \mathrm{~kb}$ )

Additional file 7: Table S5. NMR aging gene set enrichment, contains significantly affected gene sets from the proteomic analysis. (XLSX $47 \mathrm{~kb}$ )

Additional file 8: Table S6. Comparison of cross-species and NMR aging dataset for proteomic and transcriptomic data. (XLS 2175 kb)

Additional file 9: Table S7. Human aging dataset, contains differential expression analysis for proteomic data for human liver samples from young and old donors. (XLS $1778 \mathrm{~kb}$ )

Additional file 10: Figure S3. Examples of enzymes involved in fatty acid beta-oxidation and xenobiotic metabolism that decrease during aging in human liver. (A) Additional examples of enzymes involved in lipid metabolism decreasing during aging in human liver. (B) Detoxifying enzymes decreasing during aging in both NMR and humans. Only significantly affected genes are shown; cut offs: NMR aging, combined $q<0.05$; human proteome aging $q<0.1$; n.s. $=$ no significant cases detected. Related to Fig. 4. (PDF $386 \mathrm{~kb})$

Additional file 11: Figure S4. SULT1C2 decreases with aging in NMR. Related to Fig. 5. (PDF $328 \mathrm{~kb}$ )

\section{Acknowledgements}

We thank the staff members from Stockholm Zoo Skansen for providing old NMRs, Veronika Geißler and other members of NCT tissue bank for their support, and Stefan Pietsch for the support with the bioinformatics analysis. We gratefully acknowledge support from the FLI proteomics, sequencing, and bioinformatics core facilities. MP acknowledges funding from Deutsche Forschungsgemeinschaft Award Number PL 173/8-1 and Leibniz-Gemeinschaft through the Senatsausschusswettbewerb Award Number: SAW-2012-FLI-2 (together with TH). ME acknowledges funding from Deutsche Forschungsgemeinschaft Award Number GRK1715. SS acknowledges funding from Deutsche Forschungsgemeinschaft Award Number SFB/TR209 (Project B04). The FLI is a member of the Leibniz Association and is financially supported by the Federal Government of Germany and the State of Thuringia.

\section{Availability of data and materials}

RNA-seq data of GP used in de novo transcriptome assembly for cross-species comparisons were generated by [34] and are available at the Sequence Read Archive (SRP104222). RNA-seq data for NMR aging and cross-species comparisons were generated by $[2,34]$ and are available in the Gene Expression Omnibus repository (GSE98744). The corresponding gene annotation for NMR and GP are available as a gff3-file (ftp://genome.leibniz-fli.de/pub/nmr2017/). The mass spectrometry proteomics data have been deposited to the ProteomeXchange Consortium via the PRIDE [89] partner repository with the dataset identifier PXD008720.

\section{Authors' contributions}

$I H, E C, S H$, and SNS performed the experiments and analyzed the data; MB, $A S, K S, N R$, and $K H$ analyzed the data; MP, TH, AO, SS, JMK, KS, EC, and ME designed the experiments; MP, TH, and $\mathrm{AO}$ oversaw the project and wrote the manuscript. All authors read and approved the final manuscript.

\section{Competing interests}

The authors declare that they have no competing interests.

\section{Publisher's Note}

Springer Nature remains neutral with regard to jurisdictional claims in published maps and institutional affiliations.

\section{Author details}

${ }^{1}$ Leibniz Institute on Aging - Fritz Lipmann Institute (FLI), Jena, Germany. ${ }^{2}$ Institute of Anesthesiological Pathophysiology and Process Engineering, University of Ulm, Ulm, Germany. ${ }^{3}$ Leibniz Institute for Zoo and Wildlife Research (IZW), Berlin, Germany. ${ }^{4}$ Structural and Computational Biology Unit, European Molecular Biology Laboratory, Heidelberg, Germany. ${ }^{5}$ Institute of Pathology, University Medicine Greifswald, Greifswald, Germany.

Received: 21 March 2018 Accepted: 28 June 2018

Published online: 02 August 2018

\section{References}

1. Fushan AA, Turanov AA, Lee S-G, Kim EB, Lobanov AV, Yim SH, et al. Gene expression defines natural changes in mammalian lifespan. Aging Cell. 2015; 14:352-65. https://doi.org/10.1111/acel.12283.

2. Sahm A, Bens M, Szafranski K, Holtze S, Groth M, Görlach M, et al. Long-lived rodents reveal signatures of positive selection in genes associated with lifespan. PLoS Genet. 2018;14:e1007272. https://doi.org/10.1371/journal. pgen.1007272.

3. Lewis KN, Mele J, Hornsby PJ, Buffenstein R. Stress resistance in the naked mole-rat: the bare essentials - a mini-review. Gerontology. 2012;58:453-62. https://doi.org/10.1159/000335966.

4. Skulachev VP, Holtze S, Vyssokikh MY, Bakeeva LE, Skulachev MV, Markov AV, et al. Neoteny, Prolongation of Youth: From Naked Mole Rats to "Naked Apes" (Humans). Physiol Rev. 2017;97:699-720. https://doi.org/10.1152/ physrev.00040.2015

5. Keane M, Craig T, Alföldi J, Berlin AM, Johnson J, Seluanov A, et al. The Naked Mole Rat Genome Resource: facilitating analyses of cancer and longevity-related adaptations. Bioinformatics. 2014;30:3558-60. https://doi. org/10.1093/bioinformatics/btu579.

6. Kim EB, Fang X, Fushan AA, Huang Z, Lobanov AV, Han L, et al. Genome sequencing reveals insights into physiology and longevity of the naked mole rat. Nature. 2011;479:223-7. https://doi.org/10.1038/nature10533. 
7. Fang X, Seim I, Huang Z, Gerashchenko MV, Xiong Z, Turanov AA, et al. Adaptations to a Subterranean Environment and Longevity Revealed by the Analysis of Mole Rat Genomes. Cell Rep. 2014;8:1354-64. https://doi.org/10. 1016/j.celrep.2014.07.030.

8. Yu C, Li Y, Holmes A, Szafranski K, Faulkes CG, Coen CW, et al. RNA sequencing reveals differential expression of mitochondrial and oxidation reduction genes in the long-lived naked mole-rat when compared to mice. PLoS One. 2011;6:e26729. https://doi.org/10.1371/journal.pone.0026729.

9. Pérez VI, Buffenstein R, Masamsetti V, Leonard S, Salmon AB, Mele J, et al. Protein stability and resistance to oxidative stress are determinants of longevity in the longest-living rodent, the naked mole-rat. Proc Natl Acad Sci U S A. 2009;106:3059-64. https://doi.org/10.1073/pnas.0809620106.

10. Rodriguez KA, Edrey YH, Osmulski P, Gaczynska M, Buffenstein R. Altered composition of liver proteasome assemblies contributes to enhanced proteasome activity in the exceptionally long-lived naked mole-rat. PLoS One. 2012;7:e35890. https://doi.org/10.1371/journal.pone.0035890.

11. Buffenstein R. Negligible senescence in the longest living rodent, the naked mole-rat: insights from a successfully aging species. J Comp Physiol B. 2008; 178:439-45. https://doi.org/10.1007/s00360-007-0237-5.

12. Zhao Y, Tyshkovskiy A, Muñoz-Espín D, Tian X, Serrano M, de Magalhaes JP, et al. Naked mole rats can undergo developmental, oncogene-induced and DNA damage-induced cellular senescence. Proc Natl Acad Sci. 2018;115: 1801-6. https://doi.org/10.1073/pnas.1721160115.

13. Lewis KN, Wason E, Edrey YH, Kristan DM, Nevo E, Buffenstein R. Regulation of Nrf2 signaling and longevity in naturally long-lived rodents. Proc Natl Acad Sci U S A. 2015;112:3722-7. https://doi.org/10.1073/pnas.1417566112.

14. Tian X, Azpurua J, Hine C, Vaidya A, Myakishev-Rempel M, Ablaeva J, et al. High-molecular-mass hyaluronan mediates the cancer resistance of the naked mole rat. Nature. 2013;499:346-9. https://doi.org/10.1038/nature12234.

15. Andziak B, O'Connor TP, Qi W, DeWaal EM, Pierce A, Chaudhuri AR, et al. High oxidative damage levels in the longest-living rodent, the naked mole-rat. Aging Cell. 2006;5:463-71. https://doi.org/10.1111/j.1474-9726.2006.00237.x.

16. Holtze S, Eldarov CM, Vays VB, Vangeli IM, Vysokikh MY, Bakeeva LE, et al. Study of age-dependent structural and functional changes of mitochondria in skeletal muscles and heart of naked mole rats (Heterocephalus glaber). Biochem. 2016:81:1429-37. https://doi.org/10.1134/S000629791612004X.

17. Lewis KN, Andziak B, Yang T, Buffenstein R. The naked mole-rat response to oxidative stress: just deal with it. Antioxid Redox Signal. 2013;19:1388-99. https://doi.org/10.1089/ars.2012.4911.

18. Finkel T. The metabolic regulation of aging. Nat Med. 2015;21:1416-23. https://doi.org/10.1038/nm.3998.

19. Fontana L, Partridge L. Promoting Health and Longevity through Diet: From Model Organisms to Humans. Cell. 2015;161:106-18. https://doi.org/10.1016/ j.cell.2015.02.020.

20. Davinelli S, Willcox DC, Scapagnini G. Extending healthy ageing: nutrient sensitive pathway and centenarian population. Immun Ageing. 2012;9:9 https://doi.org/10.1186/1742-4933-9-9.

21. Sahm A, Bens M, Platzer M, Cellerino A. Parallel evolution of genes controlling mitonuclear balance in short-lived annual fishes. Aging Cell. 2017;16:488-96. https://doi.org/10.1111/acel.12577.

22. Houtkooper RH, Mouchiroud L, Ryu D, Moullan N, Katsyuba E, Knott G, et al. Mitonuclear protein imbalance as a conserved longevity mechanism. Nature. 2013;497:451-7. https://doi.org/10.1038/nature12188.

23. Brandt T, Mourier A, Tain LS, Partridge L, Larsson N-G, Kühlbrandt W. Changes of mitochondrial ultrastructure and function during ageing in mice and Drosophila. Elife. 2017;6 https://doi.org/10.7554/eLife.24662.

24. Park TJ, Reznick J, Peterson BL, Blass G, Omerbašić D, Bennett NC, et al. Fructose-driven glycolysis supports anoxia resistance in the naked mole-rat. Science. 2017;356:307-11. https://doi.org/10.1126/science.aab3896.

25. Fraser HB. Gene expression drives local adaptation in humans. Genome Res. 2013;23:1089-96. https://doi.org/10.1101/gr.152710.112

26. Ori A, Toyama BH, Harris MS, Bock T, Iskar M, Bork P, et al. Integrated Transcriptome and Proteome Analyses Reveal Organ-Specific Proteome Deterioration in Old Rats. Cell Syst. 2015;1:224-37. https://doi.org/10.1016/j. cels.2015.08.012

27. Tacutu R, Thornton D, Johnson E, Budovsky A, Barardo DI, Craig T, et al. Human Ageing Genomic Resources: new and updated databases. Nucleic Acids Res. 2018;46:D1083-90. https://doi.org/10.1093/nar/gkx1042.

28. Schwanhäusser B, Busse D, Li N, Dittmar G, Schuchhardt J, Wolf J, et al. Global quantification of mammalian gene expression control. Nature. 2011; 473:337-42. https://doi.org/10.1038/nature10098.
29. Vogel C, Marcotte EM. Insights into the regulation of protein abundance from proteomic and transcriptomic analyses. Nat Rev Genet. 2012;13:227-32. https://doi.org/10.1038/nrg3185.

30. Malhotra D, Portales-Casamar E, Singh A, Srivastava S, Arenillas D, Happel C, et al. Global mapping of binding sites for Nrf2 identifies novel targets in cell survival response through ChIP-Seq profiling and network analysis. Nucleic Acids Res. 2010;38:5718-34. https://doi.org/10.1093/nar/gkq212.

31. Nyström T, Yang J, Molin M. Peroxiredoxins, gerontogenes linking aging to genome instability and cancer. Genes Dev. 2012;26:2001-8. https://doi.org/ 10.1101/gad.200006.112.

32. Hanzén S, Vielfort K, Yang J, Roger F, Andersson V, Zamarbide-Forés S, et al. Lifespan Control by Redox-Dependent Recruitment of Chaperones to Misfolded Proteins. Cell. 2016;166:140-51. https://doi.org/10.1016/j.cell.2016.05.006.

33. Biteau B, Karpac J, Supoyo S, DeGennaro M, Lehmann R, Jasper H. Lifespan extension by preserving proliferative homeostasis in Drosophila. PLoS Genet. 2010;6:1-15. https://doi.org/10.1371/journal.pgen.1001159.

34. Bens M, Szafranski K, Holtze S, Sahm A, Groth M, Kestler HA, et al. Naked mole-rat transcriptome signatures of socially-suppressed sexual maturation and links of reproduction to aging. BMC Biol. 2018, in press; https://doi.org/ 10.1186/s12915-018-0546-z.

35. Erol A. The Functions of PPARs in Aging and Longevity. PPAR Res. 2007; 2007:39654. https://doi.org/10.1155/2007/39654.

36. Bustos V, Partridge L. Good Ol' Fat: Links between Lipid Signaling and Longevity. Trends Biochem Sci. 2017;42:812-23. https://doi.org/10.1016/j.tibs. 2017.07.001.

37. Cellerino A, Ori A. What have we learned on aging from omics studies? Semin Cell Dev Biol. 2017;70:177-89. https://doi.org/10.1016/j.semcdb.2017. 06.012 .

38. de Magalhães JP, Curado J, Church GM. Meta-analysis of age-related gene expression profiles identifies common signatures of aging. Bioinformatics. 2009;25:875-81.

39. Delire B, Lebrun V, Selvais C, Henriet P, Bertrand A, Horsmans Y, et al. Aging enhances liver fibrotic response in mice through hampering extracellular matrix remodeling. Aging (Albany NY). 2016;9:98-113. https://doi.org/10. 18632/aging.101124.

40. Buczak K, Ori A, Kirkpatrick JM, Holzer K, Dauch D, Roessler S, et al. Spatial Tissue Proteomics Quantifies Inter- and Intratumor Heterogeneity in Hepatocellular Carcinoma (HCC). Mol Cell Proteomics. 2018;17:810-25. https://doi.org/10.1074/mcp.RA117.000189.

41. Hughes CS, McConechy MK, Cochrane DR, Nazeran T, Karnezis AN, Huntsman DG, et al. Quantitative Profiling of Single Formalin Fixed Tumour Sections: proteomics for translational research. Sci Rep. 2016;6:34949. https://doi.org/10.1038/srep34949.

42. Hattori $\mathrm{K}$, Inoue M, Inoue T, Arai H, Tamura H. A novel sulfotransferase abundantly expressed in the dauer larvae of Caenorhabditis elegans. J Biochem. 2006;139:355-62. https://doi.org/10.1093/jb/mvj041.

43. Fielenbach $N$, Antebi A. C. elegans dauer formation and the molecular basis of plasticity. Genes Dev. 2008;22:2149-65. https://doi.org/10.1101/ gad.1701508.

44. Murphy CT, McCarroll SA, Bargmann Cl, Fraser A, Kamath RS, Ahringer J, et al. Genes that act downstream of DAF-16 to influence the lifespan of Caenorhabditis elegans. Nature. 2003;424:277-83. https://doi.org/10.1038/ nature01789.

45. MacRae SL, Croken MM, Calder RB, Aliper A, Milholland B, White RR, et al. DNA repair in species with extreme lifespan differences. Aging (Albany NY). 2015;7:1171-84. https://doi.org/10.18632/aging.100866.

46. Schmidt H, Malik A, Bicker A, Poetzsch G, Avivi A, Shams I, et al. Hypoxia tolerance, longevity and cancer-resistance in the mole rat Spalax - A liver transcriptomics approach. Sci Rep. 2017;7:1-13. https://doi.org/10.1038/ s41598-017-13905-z.

47. McNab BK. The Metabolism of Fossorial Rodents: A Study of Convergence. Ecology. 1966;47:712-33.

48. Buffenstein R, Yahav S. The effect of diet on microfaunal population and function in the caecum of a subterranean naked mole-rat. Heterocephalus glaber. Br J Nutr. 1991;65:249-58. https://doi.org/10. 1016/0306-4565(91)90030-6.

49. Holtze S, Braude S, Lemma A, Koch R, Morhart M, Szafranski K, et al. The microenvironment of naked mole-rat burrows in East Africa. Afr J Ecol. 2017;

50. Keil G, Cummings $E$, de Magalhães JP. Being cool: how body temperature influences ageing and longevity. Biogerontology. 2015;16:383-97. https:// doi.org/10.1007/s10522-015-9571-2. 
51. Larson J, Park TJ. Extreme hypoxia tolerance of naked mole-rat brain. Neuroreport. 2009:20:1634-7. https://doi.org/10.1097/WNR. Ob013e32833370cf.

52. White RR, Milholland B, MacRae SL, Lin M, Zheng D, Vijg J. Comprehensive transcriptional landscape of aging mouse liver. BMC Genomics. 2015;16:899. https://doi.org/10.1186/s12864-015-2061-8.

53. Beltrán-Sánchez H, Finch C. Age is just a number. Elife. 2018;7 https://doi. org/10.7554/eLife.34427.

54. Durieux J, Wolff S, Dillin A. The cell-non-autonomous nature of electron transport chain-mediated longevity. Cell. 2011;144:79-91. https://doi.org/10. 1016/j.cell.2010.12.016

55. Baumgart M, Priebe S, Groth M, Hartmann N, Menzel U, Pandolfini L, et al. Longitudinal RNA-seq analysis of vertebrate aging identifies mitochondrial complex i as a small-molecule-sensitive modifier of lifespan. Cell Syst. 2016; 2:122-32.

56. Miwa S, Jow H, Baty K, Johnson A, Czapiewski R, Saretzki G, et al. Low abundance of the matrix arm of complex I in mitochondria predicts longevity in mice. Nat Commun. 2014;5:3837. https://doi.org/10.1038/ ncomms4837.

57. Sgarbi G, Matarrese P, Pinti M, Lanzarini C, Ascione B, Gibellini L, et al. Mitochondria hyperfusion and elevated autophagic activity are key mechanisms for cellular bioenergetic preservation in centenarians. Aging (Albany NY). 2014;6:296-310. https://doi.org/10.18632/aging.100654.

58. Sengupta S, Peterson TR, Laplante M, Oh S, Sabatini DM. mTORC1 controls fasting-induced ketogenesis and its modulation by ageing. Nature. 2010; 468:1100-4. https://doi.org/10.1038/nature09584.

59. Luis NM, Wang L, Ortega M, Deng H, Katewa SD, Li PW-L, et al. Intestinal IRE1 Is Required for Increased Triglyceride Metabolism and Longer Lifespan under Dietary Restriction. Cell Rep. 2016;17:1207-16. https://doi.org/10.1016/ j.celrep.2016.10.003.

60. Mitchell SJ, Madrigal-Matute J, Scheibye-Knudsen M, Fang E, Aon M, González-Reyes JA, et al. Effects of Sex, Strain, and Energy Intake on Hallmarks of Aging in Mice. Cell Metab. 2016;23:1093-112. https://doi.org/ 10.1016/j.cmet.2016.05.027.

61. Hahn O, Grönke S, Stubbs TM, Ficz G, Hendrich O, Krueger F, et al. Dietary restriction protects from age-associated DNA methylation and induces epigenetic reprogramming of lipid metabolism. Genome Biol. 2017;18:56. https://doi.org/10.1186/s13059-017-1187-1.

62. Kim HE, Grant AR, Simic MS, Kohnz RA, Nomura DK, Durieux J, et al. Lipid Biosynthesis Coordinates a Mitochondrial-to-Cytosolic Stress Response. Cell. 2016;166:1539-1552.e16. https://doi.org/10.1016/j.cell.2016.08.027.

63. Weir HJ, Yao P, Huynh FK, Escoubas CC, Goncalves RL, Burkewitz K, et al. Dietary Restriction and AMPK Increase Lifespan via Mitochondrial Network and Peroxisome Remodeling. Cell Metab. 2017;26:884-896.e5. https://doi. org/10.1016/j.cmet.2017.09.024.

64. Toth M, Tchernof A. Lipid metabolism in the elderly. Eur J Clin Nutr. 2000;54: S121-5. https://doi.org/10.1038/sj.ejcn.1601033.

65. Solomon TPJ, Marchetti CM, Krishnan RK, Gonzalez F, Kirwan JP. Effects of aging on basal fat oxidation in obese humans. Metabolism. 2008;57:1141-7. https://doi.org/10.1016/j.metabol.2008.03.021.

66. St-Onge M-P, Gallagher D. Body composition changes with aging: the cause or the result of alterations in metabolic rate and macronutrient oxidation? Nutrition. 2010;26:152-5. https://doi.org/10.1016/j.nut.2009.07.004.

67. Franceschi C, Campisi J. Chronic Inflammation (Inflammaging) and Its Potential Contribution to Age-Associated Diseases. Journals Gerontol Ser A Biol Sci Med Sci. 2014;69(Suppl 1):S4-9. https://doi.org/10.1093/gerona/glu057.

68. Done AJ, Gage MJ, Nieto NC, Traustadóttir T. Exercise-induced Nrf2-signaling is impaired in aging. Free Radic Biol Med. 2016;96:130-8. https:/doi.org/10.1016/ j.freeradbiomed.2016.04.024.

69. Safdar A, de Beer J, Tarnopolsky MA. Dysfunctional Nrf2-Keap1 redox signaling in skeletal muscle of the sedentary old. Free Radic Biol Med. 2010; 49:1487-93. https://doi.org/10.1016/j.freeradbiomed.2010.08.010.

70. Buffenstein R, Jarvis JUM. The Naked Mole Rat-A New Record for the Oldest Living Rodent. Sci Aging Knowl Environ. 2002;2002:pe7. https://doi.org/10. 1126/sageke.2002.21.pe7.

71. Dammann P, Šumbera R, Maßmann C, Scherag A, Burda H. Extended Longevity of Reproductives Appears to be Common in Fukomys Mole-Rats (Rodentia, Bathyergidae). PLoS One. 2011;6:e18757. https://doi.org/10.1371/ journal.pone.0018757.

72. Dammann $\mathrm{P}, \mathrm{Burda} \mathrm{H}$. Sexual activity and reproduction delay ageing in a mammal. Curr Biol. 2006;16:R117-8. https://doi.org/10.1016/j.cub.2006.02.012.
73. Bens M, Sahm A, Groth M, Jahn N, Morhart M, Holtze S, et al. FRAMA: from RNA-seq data to annotated mRNA assemblies. BMC Genomics. 2016;17:54. https://doi.org/10.1186/s12864-015-2349-8.

74. Sahm A, Bens M, Platzer M, Szafranski K. PosiGene: automated and easy-touse pipeline for genome-wide detection of positively selected genes. Nucleic Acids Res. 2017;45:e100-0. https://doi.org/10.1093/nar/gkx179.

75. Hughes CS, Foehr S, Garfield DA, Furlong EE, Steinmetz LM, Krijgsveld J. Ultrasensitive proteome analysis using paramagnetic bead technology. Mol Syst Biol. 2014;10:757. https://doi.org/10.15252/msb.20145625.

76. Cox J, Neuhauser N, Michalski A, Scheltema RA, Olsen JV, Mann M. Andromeda: A Peptide Search Engine Integrated into the MaxQuant Environment. J Proteome Res. 2011;10:1794-805. https://doi.org/10.1021/ pr101065j.

77. Cox J, Mann M. MaxQuant enables high peptide identification rates, individualized p.p.b.-range mass accuracies and proteome-wide protein quantification. Nat Biotechnol. 2008;26:1367-72. https://doi.org/10.1038/nbt.1511.

78. Elias JE, Gygi SP. Target-decoy search strategy for increased confidence in large-scale protein identifications by mass spectrometry. Nat Methods. 2007; 4:207-14. https://doi.org/10.1038/nmeth1019.

79. Ritchie ME, Phipson B, Wu D, Hu Y, Law CW, Shi W, et al. limma powers differential expression analyses for RNA-sequencing and microarray studies. Nucleic Acids Res. 2015;43:e47-7. https://doi.org/10.1093/nar/gkv007.

80. Benjamini Y, Hochberg Y. Controlling the False Discovery Rate: A Practical and Powerful Approach to Multiple Testing. Journal of the Royal Statistical Society. Series B (Methodological). 1995;57:289-300. https://doi.org/10.2307/ 2346101.

81. Brosch M, Yu L, Hubbard T, Choudhary J. Accurate and sensitive peptide identification with Mascot Percolator. J Proteome Res. 2009;8:3176-81.

82. Gatto L, Lilley KS. MSnbase-an R/Bioconductor package for isobaric tagged mass spectrometry data visualization, processing and quantitation. Bioinformatics. 2012;28:288-9. https://doi.org/10.1093/bioinformatics/btr645.

83. Huber W, von Heydebreck A, Sultmann H, Poustka A, Vingron M. Variance stabilization applied to microarray data calibration and to the quantification of differential expression. Bioinformatics. 2002;18(Suppl 1):S96-104. https:// doi.org/10.1093/bioinformatics/18.suppl_1.S96

84. Bruderer R, Bernhardt OM, Gandhi T, Miladinović SM, Cheng L-Y, Messner S, et al. Extending the limits of quantitative proteome profiling with dataindependent acquisition and application to acetaminophen-treated threedimensional liver microtissues. Mol Cell Proteomics. 2015;14:1400-10. https://doi.org/10.1074/mcp.M114.044305.

85. Storey JD. A direct approach to false discovery rates. J R Stat Soc Ser B (Statistical Methodol). 2002;64:479-98. https://doi.org/10.1111/1467-9868.00346.

86. Luo W, Friedman MS, Shedden K, Hankenson KD, Woolf PJ. GAGE: generally applicable gene set enrichment for pathway analysis. BMC Bioinformatics. 2009;10:161. https://doi.org/10.1186/1471-2105-10-161.

87. Subramanian A, Tamayo P, Mootha VK, Mukherjee S, Ebert BL, Gillette MA, et al. Gene set enrichment analysis: a knowledge-based approach for interpreting genome-wide expression profiles. Proc Natl Acad Sci U S A 2005;102:15545-50. https://doi.org/10.1073/pnas.0506580102.

88. Eden E, Navon R, Steinfeld I, Lipson D, Yakhini Z. GOrilla: a tool for discovery and visualization of enriched $\mathrm{GO}$ terms in ranked gene lists. BMC Bioinformatics. 2009;10:48. https://doi.org/10.1186/1471-2105-10-48.

89. Vizcaíno JA, Csordas A, Del-Toro N, Dianes JA, Griss J, Lavidas I, et al. 2016 update of the PRIDE database and its related tools. Nucleic Acids Res. 2016; 44:D447-56. https://doi.org/10.1093/nar/gkv1145.

\section{Ready to submit your research? Choose BMC and benefit from:}

- fast, convenient online submission

- thorough peer review by experienced researchers in your field

- rapid publication on acceptance

- support for research data, including large and complex data types

- gold Open Access which fosters wider collaboration and increased citations

- maximum visibility for your research: over $100 \mathrm{M}$ website views per year

At BMC, research is always in progress.

Learn more biomedcentral.com/submissions 